University of Wollongong

Research Online

Faculty of Business - Papers (Archive)

Faculty of Business and Law

$1-1-2016$

Investing in skill and searching for coworkers: endogenous participation in a matching market

Chris Bidner

Simon Fraser University

Guillaume Roger

University of Sydney, groger@uow.edu.au

Jessica Moses

Deloitte

Follow this and additional works at: https://ro.uow.edu.au/buspapers

Part of the Business Commons

Research Online is the open access institutional repository for the University of Wollongong. For further information contact the UOW Library: research-pubs@uow.edu.au 


\title{
Investing in skill and searching for coworkers: endogenous participation in a matching market
}

\author{
Abstract \\ We demonstrate how search frictions have important yet subtle implications for participation in a skilled \\ labor market by studying a model in which agents invest in skill prior to searching for coworkers. Search \\ frictions induce the existence of acceptance-constrained equilibria, whereby matching concerns-as \\ opposed to investment costs-dissuade the marginal agent from investing and participating in the skilled \\ matching market. Such equilibria are robust, relevant, and have comparative static properties that \\ contrast sharply with the intuitive properties arising in a benchmark static setting. We consider an \\ extension with separate matching "marketplaces," and show that our main results continue to hold. \\ Disciplines \\ Business

\section{Publication Details} \\ Bidner, C., Roger, G. \& Moses, J. (2016). Investing in skill and searching for coworkers: endogenous \\ participation in a matching market. American Economic Journal: Microeconomics, 8 (1), 166-202.
}




\title{
Investing in Skill and Searching for Coworkers: Endogenous Participation in a Matching Market*
}

\author{
Chris Bidner $^{\dagger} \quad$ Guillaume Roger ${ }^{\ddagger} \quad$ Jessica Moses $\S$
}

November 26, 2014

\begin{abstract}
We demonstrate how search frictions have important yet subtle implications for participation in a skilled labor market by studying a model in which agents invest in skill prior to searching for coworkers. Search frictions induce the existence of acceptance constrained equilibria, whereby matching concerns-as opposed to investment costs-dissuade the marginal agent from investing and participating in the skilled matching market. Such equilibria are robust, relevant, and have comparative static properties that contrast sharply with the intuitive properties arising in a benchmark static setting. We consider an extension with separate matching 'marketplaces', and show that our main results continue to hold.
\end{abstract}

Keywords: matching, search frictions, pre-match investment.

JEL Classification: D83, J24, D02.

\footnotetext{
*We would like to thank Ken Burdett, Nicholas Jacquet, Mike Peters, Kunal Sengupta, Andy Skrzypacz, Randy Wright, three anonymous referees as well as seminar audiences at the 2014 Singapore Search and Matching Workshop, the 2013 Asian Meeting of the Econometric Society, the 2013 North American Meeting of the Econometric Society, the University of New South Wales, University of Tasmania, University of Adelaide, the University of Sydney, and University of Queensland, for useful comments. All errors are ours.

${ }^{\dagger}$ Corresponding Author. Department of Economics, Simon Fraser University (cbidner@sfu.ca)

${ }^{\ddagger}$ School of Economics, The University of Sydney (guillaume.roger72@gmail.com)

${ }^{\S}$ Deloitte-Access Economics (jesmoses@deloitte.com.au)
} 


\section{Introduction}

An economy's prospects are strongly tied to its capacity to cultivate a high-skilled workforce. ${ }^{1}$ In light of this, it is clearly important to understand the incentives of workers to participate in the skilled labor market. We examine such incentives in an environment in which a skilled worker's productivity is influenced by who they work with, and where the process of finding coworkers is subject to search frictions. ${ }^{2}$

Far from simply adding to the effective cost of investing in skill, search frictions impinge in subtle ways upon incentives to participate in the skilled labor market. Search has a clear impact on incentives insofar as frictions influence the expected length of time required to find an acceptable match. Beyond this, the evaluation of potential matches is inherently a two-sided process and as such search introduces a concern among workers as to who, if anyone, is willing to form a productive match with them in equilibrium. This observation leads to the emergence of a class of equilibria, which we call acceptance constrained equilibria, in which the marginal participant is determined by matching concerns and not by the cost of investing in skill. In such equilibria, participation is strictly

\footnotetext{
${ }^{1}$ In a recent speech (April 2, 2014), Barack Obama outlines how "Opportunity means training more Americans for the skills needed to fill [good] jobs ... Opportunity means guaranteeing every young people access to a world-class education ... And it means making college more affordable" (http://www.whitehouse.gov/the-pressoffice/2014/04/02/remarks-president-minimum-wage-ann-arbor-mi). Commentators also point to the social and business implications of skill disparities across workers. In an article examining the impact of technological change, The Economist ("The Onrushing Wave," Jan 18, 2014) concludes that "society may find itself sorely tested if, as seems possible, growth and innovation deliver handsome gains to the skilled, while the rest cling to dwindling employment opportunities at stagnant wages", and Dominic Barton, managing director of McKinsey \& Company, writes in The Economist ("Young, Gifted, and Slack", November 21, 2012), "One of the biggest problems facing the world in 2013 is the prolonged-and seemingly intractable-crisis of youth unemployment. Put simply, too many young people lack employable skills in a world that has too few skilled workers."

${ }^{2}$ There are a variety of reasons why a worker's productivity would be sensitive to who they work with. First, technological advances in the workplace, most notably computerization, have led to employment being increasing concentrated in jobs that require 'non-routine' tasks (e.g. see Autor et al. (2003)). The most important among such tasks are those classified as requiring complex communication and expert thinking (Levy and Murnane (2004), Murnane and Levy (1996)), both of which are naturally social in nature; they are more effectively executed in the presence of coworkers that are themselves effective communicators and experts upon which informal collaboration, brainstorming, etc. can be relied upon. Second, innovations in human resource management stress practices such as establishing problem-solving teams, cross-training for multiple jobs, and labor-management communication procedures (Ichniowski and Shaw (2003)). Empirical evidence linking such practices to greater social capital within the workplace is presented in Gant et al. (2002) and Drago and Garvey (1998). Third, and most direct, the existence of human capital externalities has been argued to be an important ingredient in understanding cross-country income differences (e.g. Lucas (1988)) and have been shown to be important empirically (e.g. Moretti (2004), although see Acemoglu and Angrist (1999) and Sand (2013)). See Bidner (2014) for an elaboration on these points.
} 
preferred by the marginal participator. Non-participators, even those that are arbitrarily similar to the marginal participator, are dissuaded from participating because they would face matching prospects that are dramatically worse than those facing the marginal participator. Specifically, they are not accepted by any other market participant. We explore the properties of these equilibria from both a theoretical and policy perspective.

In more formal terms, our model is one in which heterogeneous agents must bear a cost in order to participate in a frictional one-sided matching market. Agents are heterogeneous with respect to their skill potential, which materializes into skill only once the agent makes a costly investment, e.g. completes higher education. This skill is useful in the skilled sector, where workers produce in the presence of a coworker. Each skilled agent produces and consumes their own individual output, but that output exhibits skill complementarity. Finding a coworker in the skilled labor market takes time. Once two agents encounter one another, both sides decide whether to leave the market and produce with the other agent, or to return to the market to continue the search for a coworker. Agents that do not make the investment work in the unskilled sector and earn a flat wage (normalized to zero).

Since workers consume their own output, matching involves non-transferable utility (see section 5 for a discussion). This, along with multiplicative separability, implies block segregation in equilibrium (see Smith (2006)). Participating agents are partitioned into 'classes' and only end up matching with agents from their class. Given this, there is scope for coordination problems whereby would-be members of a class do not participate because no one else in the class does. In the most extreme case no agent participates because no other agent does. Even if equilibria such as this have interesting properties, in many cases they are not compelling. To formalize this claim we introduce a notion of robustness. In short, robustness requires that there are no profitable joint deviations for groups of arbitrarily small size. ${ }^{3}$ When the investment cost is low for instance, the 'no participation' equilibrium is not robust: an arbitrarily small group consisting of agents with the highest levels of potential skill always profit by jointly investing. To be sure, focusing on robust equilibria imposes discipline on our analysis since it makes the task of identifying 'interesting' equilibria more difficult.

Equilibria involve a cut-off skill potential (which we identify with 'type'), whereby an agent

\footnotetext{
${ }^{3}$ Mailath et al. (2013b) discuss a coordination problem within the context of prematch investment (in a static setting), but the nature of this problem is qualitatively distinct from the one highlighted here. In that paper agents may make low investments (e.g. zero) because potential partners make low investments. Our coordination problem stems from the participation decision rather than the magnitude of investment. The type of coordination problem discussed in Mailath et al. (2013b) is not robust according to our criteria since it can be overcome if an arbitrarily small measure of agents change their behavior (investment choice). Key for us is the issue that sufficiently many others need to change behavior in order to overcome the coordination problem.
} 
participates if their type is above the cut-off. Furthermore, all equilibria belong to one of two broad classes. The first one, which we call cost constrained equilibria (CCE), have the cut-off type determined by the investment cost. Marginally lower types would enjoy matching prospects similar to the cut-off type, but are dissuaded from investing because it is too costly. The second class is the acceptance constrained equilibria (ACE) described earlier. A robust equilibrium always exists, but it needs not be unique. Both classes of equilibria are relevant in the sense that there is a nontrivial set of parameters for which the unique robust equilibrium is cost constrained, and similarly for acceptance constrained. When there are multiple robust equilibria, it can be that they are all acceptance constrained or there is a coexistence of acceptance constrained and cost constrained equilibria.

To highlight the impact of the search problem we begin with a static benchmark in which investing agents are randomly matched. This is useful to show that cost constrained equilibria (of the dynamic model) have properties that are broadly similar to equilibria in the static benchmark. Acceptance constrained equilibria are qualitatively different. First, ACE possess counter-intuitive (local) comparative static properties; e.g. participation is insensitive to investment cost, and is increasing in search frictions. Second, the robust coordination problem that underlies the existence of multiple ACE introduces the possibility that some ACE exhibit under-investment, in contrast to the static benchmark. Finally, explicitly considering the participation decision places restrictions on the set of agents predicted to participate; it is not the case that any type can be supported as the marginal entrant in a robust equilibrium for some investment cost.

If the acceptance concern in the matching market is responsible for generating the coordination problem, it is perhaps natural to consider allowing separate matching markets to form. To this end we consider an extension based on Jacquet and Tan (2007) in which each class of agent separates into their own matching market. The key insights derived in the main analysis persist in this environment. Specifically, robust ACE and CCE continue to exist and have the same comparative static properties. This extension also highlights further benefits of such market segmentation, over and above those identified by Jacquet and Tan (2007). Specifically, the multiplicity of robust equilibria disappears. ${ }^{4}$

In recognizing that agents take conscious efforts to improve their matching-related outcomes, our model is related to a literature on pre-match investment (e.g. Nöldeke and Samuelson (2014), Mailath et al. (2013a), Mailath et al. (2013b), Hatfield et al. (2014), Peters and Siow (2002), Cole et al. (2001), Iyigun and Walsh (2007), Zhang (1994), Anderson and Bidner (2013), Gall et al. (2012), Gall et al. (2006), Booth and Coles (2010), and Felli and Roberts (2002)). The feature

\footnotetext{
${ }^{4}$ To be sure, the multiplicity disappears because having separate matching markets fundamentally changes the strategic environment, and not because it selects among the multiple equilibria that arise in the main analysis.
} 
that investment facilitates assortative matching on types makes the analysis particularly related to the smaller literature on pre-match investment with imperfect information (Hopkins (2012), Hoppe et al. (2009), Bidner (2010), and Bidner (2014)). In contrast to these literatures, we explicitly incorporate search frictions.

The model is also related to a large literature on search and matching (e.g. Shimer and Smith (2000), Smith (2006), Burdett and Coles (1997), and Jacquet and Tan (2007)). In contrast to these papers we explicitly incorporate the skill investment decision. In this respect our paper is particularly related to models of search and matching with investment, such as Burdett and Coles (2001) and de Meza and Lockwood (2010). The fundamental difference lies in the timing, and therefore the role, of investment. They take the set of participants in the matching market as fixed and consider costly additions to skill ('pizzazz'). In contrast, we are interested in investment insofar as it affects incentives to participate in the matching market; that is, investment takes place ex ante. To focus on this we abstract from the intensive margin, although we agree that this dimension is not trivial. In appendix section D.1 we argue that our results continue to hold if allowing for a richer investment space as long as it is discrete.

In addressing how agents are sorted across markets in a matching context, the paper is related

to a literature concerned with 'match-makers'. For instance, Damiano and Li (2007) consider a static setting in which a monopolist sorts agents into different matching markets by offering a menu of entry fees. Bloch and Ryder (2000) consider a match-maker that charges a fixed fee to induce searchers to leave the decentralized search market in order to be matched centrally by the match maker.

The remaining of the paper is organized as follows. The fundamentals of the model, as well as a benchmark setting, are introduced and analyzed in section 2 . The main dynamic model is presented and analyzed in section 3. The extension to multiple matching markets is considered in section 4. We discuss the importance of our modeling assumptions in section 5, and draw conclusions in section 6 . All proofs of results presented in the text are in section B of the appendix.

\section{Model Fundamentals and Benchmark}

\section{$2.1 \quad$ Fundamentals}

We begin with a description of the model fundamentals that we use in a static benchmark. The dynamic aspects are introduced later, as required. Agents can produce in either the unskilled or the skilled sector. All agents have equal productivity in the unskilled sector, producing and consuming an output normalized to zero. An agent's productivity in the skilled sector depends on their skill 
and that of their coworker. ${ }^{5}$ Specifically, if agent $i$ has skill $s_{i}$ and has $j$ as a coworker, then $i$ produces and consumes

$$
y_{i}=s_{i} \cdot s_{j}
$$

This specification is chosen for simplicity; our main results go through as long as $y_{i}$ can be expressed as $\gamma_{1}\left(s_{i}\right) \cdot \gamma_{2}\left(s_{j}\right)$, where $\gamma_{1}$ and $\gamma_{2}$ are increasing differentiable functions (Smith (2006)). That agents consume their own output means that we assume non-transferable utility, which we discuss at some length in Section 5. To become skilled, agents must undertake an investment (e.g. a university education) at cost $c>0 .{ }^{6}$ Conditional on investing skills are heterogeneous. To model this in the most transparent manner, we endow each agent with a type, $\theta_{i} \in \Theta \equiv[0,1]$, that can be interpreted as their 'skill potential' or 'natural ability'. Let the investment choice be denoted $x_{i} \in\{0,1\}$; worker $i$ 's skill is $s_{i}=x_{i} \cdot \theta_{i}$. The distribution of types is given by $F$, with associated density $f$, which we assume to be $\log$ concave. ${ }^{7}$

\section{$2.2 \quad$ A Static Benchmark}

To set a benchmark for the results to follow, suppose that there are a continuum of workers and production is one-shot. Agents first observe their ability and decide whether to invest. Then they decide whether they want to work in the unskilled or the skilled sector. No agent would incur the costly investment if they intended to enter the unskilled sector (where such investments are unrewarded), so the payoff to entering the unskilled sector is zero. Likewise, it is never optimal to choose the skilled sector without investing - this ensures a payoff of zero. So we focus on agents who may decide to invest and enter the skilled sector. Then the payoff depends on the distribution of types that also choose the skilled sector.

Agents in the skilled sector are randomly matched together. The one-shot nature of the game implies that production with the assigned partner always occurs. As such, worker $i$ expects to produce $\bar{y}_{i}=s_{i} \cdot \bar{s}$ where $\bar{s}$ is the average skill in the skilled sector. The net expected payoff from investing for worker $i$ is $\theta_{i} \cdot \bar{s}-c$. Equilibrium must therefore involve a cut-off type, $\hat{\theta}$, such that a worker invests if and only if $\theta \geq \hat{\theta}$. The cut-off can be greater than one; then no agents invest.

\footnotetext{
${ }^{5}$ We abstract from the reality that agents may have more than one coworker. Extending the model in this direction is certainly of interest, but introduces various issues (e.g. match formation, group size, asymmetric spillovers, etc.) that are beyond the scope of this paper.

${ }^{6}$ In section D.1 we show that the assumption of a binary investment technology is for simplicity.

${ }^{7}$ See section A.1 in the appendix for the key implication of this assumption.
} 
Given the cut-off type, we have

$$
\bar{s}=\bar{s}(\hat{\theta}) \equiv \begin{cases}\mathbb{E}[\theta \mid \theta \geq \hat{\theta}] & \text { if } \hat{\theta} \leq 1 \\ 0 & \text { otherwise }\end{cases}
$$

so that the net payoff from investing for type $\theta$ is:

$$
u(\theta, \hat{\theta})=\theta \cdot \bar{s}(\hat{\theta})-c .
$$

If $\hat{\theta}^{*}$ is an equilibrium cut-off it must be that $u\left(\theta, \hat{\theta}^{*}\right) \geq 0$ for all $\theta \geq \hat{\theta}^{*}$ and $u\left(\theta, \hat{\theta}^{*}\right) \leq 0$ for all $\theta<\hat{\theta}^{*}$. Clearly if $c>1$ it is never optimal to invest (so that any $\hat{\theta}^{*}>1$ is an equilibrium cut-off). An equilibrium with positive investment exists when $c \leq 1$. Any $\hat{\theta}^{*} \leq 1$ must satisfy

$$
\hat{\theta}^{*} \cdot \mathbb{E}\left[\theta \mid \theta \geq \hat{\theta}^{*}\right]-c=0
$$

by continuity of $u$ in $\theta$. The left side is a strictly increasing and continuous function of $\hat{\theta}^{*}$ on $[0,1]$, approaching a negative value as $\hat{\theta}^{*} \rightarrow 0$ and a positive value as $\hat{\theta}^{*} \rightarrow 1$. So there is a unique solution to Equation (2.2) and therefore there exists a unique equilibrium with positive investment when $c<1$. This is not the unique equilibrium however since 'no-investment' is also an equilibrium outcome when $c<1$ due to co-ordination failure. We argue that such equilibria are uninteresting because they are not robust in sense that there exists a positive but arbitrarily small measure of non-investors that could profit from a joint deviation. Formally,

Definition 1 Consider an equilibrium with cut-off type $\hat{\theta}^{*}$, and let

$$
\tilde{\theta} \equiv \sup \left\{\theta: \theta \in \Theta, u\left(\theta, \hat{\theta}^{*}\right) \leq 0\right\} .
$$

The equilibrium is said to be "robust" if $u(\tilde{\theta}-\varepsilon, \tilde{\theta}-\varepsilon)<0$ for all $\varepsilon \in(0, \bar{\varepsilon})$ for some $\bar{\varepsilon}>0$.

In words, $\tilde{\theta}$ is the 'highest' type among those that weakly prefer to not invest. 8 This notion of robustness imposes the requirement that there do not exist profitable joint deviations for any group of arbitrarily small measure. If any group of workers were able to profitably deviate to investing, it would be most profitable for the highest types among the non-investors. Therefore it is sufficient to check that there are no profitable joint deviations for workers with types just below $\tilde{\theta}$ : i.e. $u(\tilde{\theta}-\varepsilon \mid \tilde{\theta}-\varepsilon)<0$ for all $\varepsilon$ sufficiently small.

When $c \geq 1$ 'no-investment' is a robust equilibrium outcome; the top agents do not find it profitable to invest even if they match with the highest type with certainty. For $c<1$ 'noinvestment' is not a robust equilibrium outcome; high types would profit by investing as soon as other high types invest. If $c \leq 1$, the unique equilibrium with investment is robust. ${ }^{9}$

\footnotetext{
${ }^{8}$ If the equilibrium exhibits investment, so that $\hat{\theta}^{*} \in \Theta$, then $\tilde{\theta}$ coincides with $\hat{\theta}^{*}$. The more involved definition is required in order to cover equilibrium without investment. In this case, $\tilde{\theta}$ is the highest type.

${ }^{9}$ See Results 4-6 in section A.2 of the appendix for proofs of the claims made in this paragraph.
} 
Proposition 1 There exists a unique robust equilibrium when $c<1$. This equilibrium is characterized by the unique cut-off type, $\hat{\theta}^{*} \in(0,1)$, that satisfies (2.2).

From here assume that $c<1$ and thus focus on the unique robust equilibrium to explore its properties. The equilibrium measure of participating agents is $1-F\left(\hat{\theta}^{*}\right)$.

Result 1 The measure of participating agents is strictly decreasing in the investment cost.

This result implies that participation levels can be manipulated by imposing an investment tax/subsidy. In order to get a sense of whether a planner would want to manipulate incentives, and if so in what direction, we begin by noting that a planner concerned with maximizing the average payoff will use a cut-off rule. A cut-off of $\hat{\theta}$ produces an average payoff of

$$
W(\hat{\theta}) \equiv F(\hat{\theta}) \cdot 0+(1-F(\hat{\theta})) \cdot\left[E[\theta \mid \theta \geq \hat{\theta}]^{2}-c\right]=(1-F(\hat{\theta})) \cdot\left[E[\theta \mid \theta \geq \hat{\theta}]^{2}-c\right],
$$

and the planner's optimal cut-off, denoted $\hat{\theta}^{* *}$, maximizes $W(\hat{\theta})$.

Result 2 There is over-investment in equilibrium in the sense that $\hat{\theta}^{*}<\hat{\theta}^{* *}$.

The marginal participating type does not take into account the negative externality that they impose on all other types, therefore leading to excessive entry. This standard result is exacerbated by skill complementarities in that the social value of a type's participation depends on which other types participate. Here a planner would like to impose an investment tax.

Skill complementarity implies that welfare would increase if matching could be made more assortative. This could potentially be achieved by creating separate vertically differentiated matching pools that each charge a pool-specific entry fee (e.g. as in Damiano and Li (2007)). ${ }^{10}$ However, there is no role for separate horizontally differentiated matching pools each of which differ only in name. It is impossible to achieve any degree of segregation via such pools in this static setting: anticipating that they will always be accepted by their partner, agents simply choose the pool with the highest expected worker skill. If there is any degree of segregation, there will always be a 'best' pool. As such, all agents will choose to enter that pool-thereby contradicting the existence of some degree of segregation. We return to this issue in section 4, where we show how such pools can segregate workers when the dynamic search problem is considered.

To summarize the static analysis we note (i) robust equilibrium outcomes are generically unique, (ii) there is over-investment but since participation is sensitive to investment cost, over-investment

\footnotetext{
${ }^{10}$ For instance, one could imagine a system with vertically differentiated universities, whereby institutions within an 'elite' group charge more than do those in a 'non-elite' group and whereby coworker search only occurs within separate 'elite' and 'non-elite' markets.
} 
can be resolved by imposing an appropriate (strictly positive) tax on investment. ${ }^{11}$ Furthermore, efficiency-enhancing segregation cannot be supported via horizontal differentiation. This paper asks whether these broad lessons are robust to the possibility that workers can reject partners. To do so requires embedding the problem in a dynamic setting.

\section{Dynamic Model: the Main Analysis}

The analysis is broken in two stages - the investment stage and the matching stage. In the spirit of backward induction we first analyze the matching stage for a fixed cut-off type and then the investment stage (thereby endogenizing the marginal investor). We begin by introducing the necessary elements to the dynamic setting.

Time is continuous, agents discount the future at the rate $r>0$, and the matching function exhibits constant returns to scale whereby potential partners are encountered at the Poisson rate $\alpha>0$. We follow much of the related literature (e.g. Burdett and Coles (2001)) in making the simplifying 'clone' assumption: once an agents leaves the matching market, a new one of the same type is born. We discuss this assumption in section 5, where we show that it is useful but inessential.

Strategies now involve a description of (i) whether to invest; let $x(\theta)$ denote the decision rule of type $\theta$, and (ii) which types to accept as partners; let $a\left(\theta^{\prime}, \theta\right) \in\{0,1\}$ be the acceptance decision of type $\theta$ when meeting type $\theta^{\prime}$. The strategy of type $\theta$ is summarized as $\sigma(\theta) \equiv\left\{x(\theta),\left\{a\left(\theta^{\prime}, \theta\right)\right\}_{\theta^{\prime} \in \Theta}\right\}$; let $\sigma \equiv\{\sigma(\theta)\}_{\theta \in \Theta}$ denote a strategy profile.

Let $G_{\sigma}$ denote the distribution of types in the skilled sector induced by strategy $\sigma$. The value of remaining unmatched for a type $\theta$ agent, denoted $U_{\sigma}(\theta)$, satisfies the standard Bellman ('asset value') equation:

$$
r U_{\sigma}(\theta)=\alpha \cdot \int_{0}^{1} a\left(\theta, \theta^{\prime}\right) \cdot \max \left\{Y\left(\theta, \theta^{\prime}\right)-U_{\sigma}(\theta), 0\right\} d G_{\sigma}\left(\theta^{\prime}\right)
$$

where

$$
Y\left(\theta, \theta^{\prime}\right) \equiv \theta \theta^{\prime} / r
$$

is the present value of output for type $\theta$ when matched with a type $\theta^{\prime}$ agent. Intuitively, (3.1) says that the flow value of being in the unmatched state for a type $\theta$ agent equals their expected gains from search. These expected gains arise from the formation of a mutually agreeable match and is the product of the rate at which potential partners are encountered, $\alpha$, and the expected value gain from an encounter. The type of an encountered potential partner is distributed according to

\footnotetext{
${ }^{11}$ Multiple robust equilibrium outcomes only arise when $c=1$ : it is a (robust) equilibrium for only those of the highest type to invest, and for there to be no investment.
} 
$G_{\sigma}$ and the value gain from encountering a type $\theta^{\prime}$ is $a\left(\theta, \theta^{\prime}\right) \cdot \max \left\{Y\left(\theta, \theta^{\prime}\right)-U_{\sigma}(\theta), 0\right\}$. To see why this is so, note that if a type $\theta^{\prime}$ is encountered, then no change in value is registered if they are unwilling to match (i.e. if $a\left(\theta, \theta^{\prime}\right)=0$ ). If they are willing to match, the type $\theta$ agent either chooses to switch to a matched state, enjoying a value gain of $Y\left(\theta, \theta^{\prime}\right)-U_{\sigma}(\theta)$, or to remain in the unmatched state for zero gain, depending on which gain is greater.

A strategy profile $\sigma^{*}$ is an equilibrium if for all $\theta \in \Theta$, (i) investment is optimal: $x(\theta)=1$ if and only if $U_{\sigma}(\theta)-c \geq 0$, and (ii) coworker acceptance is optimal: $a\left(\theta^{\prime}, \theta\right)=1$ if and only if $Y\left(\theta, \theta^{\prime}\right) \geq U_{\sigma}(\theta)$, where $U_{\sigma}$ satisfies (3.1) and $Y\left(\theta, \theta^{\prime}\right)$ is given by (3.2).

The first equilibrium condition is straightforward, but the second equilibrium condition plays two roles worth highlighting. The first, and more important, role is in imposing sequential rationality. Specifically, we want to rule out equilibria in which some types are dissuaded from participating because participants make non-credible threats to not accept such types as partners. The second is ruling out uninteresting equilibria (that hinge on the use of weakly dominated strategies) in which some agents do not accept a profitable match partner because the partner is similarly unwilling to accept them.

Equilibrium again involves a cut-off type, ${ }^{12} \hat{\theta}$, such that those in the skilled sector are those with

types above $\hat{\theta}$. As a result we write the distribution of types in the skilled sector as $G_{\sigma}=G(\cdot \mid \hat{\theta})$, and similarly $U_{\sigma}(\theta)=U(\theta \mid \hat{\theta}) .{ }^{13}$

\subsection{Matching Outcomes for Fixed Participation}

If we fix the cut-off type, then the resulting search and matching problem is a standard one-e.g. essentially that studied in Burdett and Coles (1997), Bloch and Ryder (2000), Jacquet and Tan (2007), and Smith (2006). In such models, it is well-known that agents segregate into 'classes' whereby the type space is partitioned, and agents only match with others in their partition. This segregation follows from the acceptance problem and since it is a central feature of the equilibrium we describe, we sketch the construction of classes here. This construction is then used to derive equilibrium payoffs.

Type $\theta$ accepts all types $\theta^{\prime}$ for which $Y\left(\theta, \theta^{\prime}\right) \geq U(\theta)$; that is, all types such that $\theta^{\prime} \geq R(\theta) \equiv$ $r U(\theta) / \theta$. Divide both sides of the asset value equation (3.1) by $\theta$,

$$
\begin{aligned}
R(\theta) & =\frac{\alpha}{r} \cdot \int_{0}^{1} a\left(\theta, \theta^{\prime}\right) \cdot \max \left\{\theta^{\prime}-R(\theta), 0\right\} \cdot d G\left(\theta^{\prime} \mid \hat{\theta}\right) \\
& =\frac{\alpha}{r} \cdot \int_{R(\theta)}^{1} a\left(\theta, \theta^{\prime}\right) \cdot\left[\theta^{\prime}-R(\theta)\right] \cdot d G\left(\theta^{\prime} \mid \hat{\theta}\right) .
\end{aligned}
$$

\footnotetext{
${ }^{12}$ For a proof, see corollary 4 in section A.3 of the Appendix.

${ }^{13}$ Under the clone assumption, we have $G(\theta \mid \hat{\theta}) \equiv[F(\theta)-F(\hat{\theta})] /[1-F(\hat{\theta})]$.
} 
This expression implicitly defines $R(\theta) .{ }^{14}$ Notice that if we knew that some type $\theta^{\dagger}$ was accepted by every other type, so that $a\left(\theta^{\dagger}, \theta^{\prime}\right)=1$ for all $\theta^{\prime}$, then this can be used in (3.3) to compute $R\left(\theta^{\dagger}\right)$. Specifically, type $\theta^{\dagger}$ is willing to accept all types above $R_{1}$, where this satisfies

$$
R_{1}=\frac{\alpha}{r} \cdot \int_{R_{1}}^{1}\left[\theta^{\prime}-R_{1}\right] \cdot d G\left(\theta^{\prime} \mid \hat{\theta}\right) .
$$

Notice that $R_{1}$ does not depend on $\theta^{\dagger}$, but rather only depends on the fact that they are accepted by all other types. ${ }^{15}$ To begin, we note that the highest type is always accepted by all others. Thus, the highest type accepts all types above $R_{1}$. But if a type above $R_{1}$ is accepted by the highest type, then they too must be accepted by all types. ${ }^{16}$ This being the case, we conclude that all types above $R_{1}$ also use a cut-off of $R_{1}$.

Unlike in models without entry (Burdett and Coles (1997), Bloch and Ryder (2000), Jacquet and Tan (2007)), we cannot yet conclude that types in $\left[R_{1}, 1\right]$ characterize the set of class-1 agents. Indeed, some types in that set may not enter the skilled sector. Specifically, if $R_{1} \leq \hat{\theta}$, then the set of "class-1" agents is $[\hat{\theta}, 1]$. On the other hand, if $R_{1}>\hat{\theta}$, the set of "class- 1 " types is $\left[R_{1}, 1\right]$. So the lower bound of class- 1 is $\theta_{1} \equiv \max \left\{R_{1}, \hat{\theta}\right\}$, and the set of class- 1 types is $\Theta_{1} \equiv\left[\theta_{1}, 1\right]$.

If $\theta_{1}=\hat{\theta}$ the description of the matching process is complete. ${ }^{17}$ Otherwise we need to describe the behaviour of types outside of $\Theta_{1}$, for which we return to (3.3). Types marginally below the class-1 cut-off, $R_{1}$, are acceptable by all others because such types become the best alternative outside of class- $1 .{ }^{18}$ These types use a cut-off $R_{2}$ such that

$$
R_{2}=\frac{\alpha}{r} \cdot \int_{R_{2}}^{R_{1}}\left[\theta^{\prime}-R_{2}\right] \cdot d G\left(\theta^{\prime} \mid \hat{\theta}\right)
$$

and a "class-2" may be formed. The lower bound for this class is $\theta_{2} \equiv \max \left\{R_{2}, \hat{\theta}\right\}$, and the set of types characterising class- 2 is $\Theta_{2} \equiv\left[\theta_{2}, \theta_{1}\right)$. This process continues in the expected way: if $R_{k-1}>\hat{\theta}$, the cut-off acceptable type for class- $k$ agents satisfies

$$
R_{k}=\frac{\alpha}{r} \cdot \int_{R_{k}}^{R_{k-1}}\left[\theta^{\prime}-R_{k}\right] \cdot d G\left(\theta^{\prime} \mid \hat{\theta}\right)
$$

and the lower bound for class- $k$ is $\theta_{k} \equiv \max \left\{R_{k}, \hat{\theta}\right\}$ so that class- $k$ is characterized by $\Theta_{k} \equiv$ $\left[\theta_{k}, \theta_{k-1}\right)$. This goes on until we come to the value of $K$ such that $R_{K} \leq \hat{\theta}$, at which point we

\footnotetext{
${ }^{14} \mathrm{~A}$ unique value of $R$ satisfies this equation since the left side starts at zero and is strictly increasing whereas the right side starts at a non-negative value and is weakly decreasing ending at zero when $R=1$.

${ }^{15}$ This arises because $y_{i}$ can be expressed as $\gamma_{1}\left(s_{i}\right) \cdot \gamma_{2}\left(s_{j}\right)$, where $\gamma_{1}$ and $\gamma_{2}$ are increasing differentiable functions (Smith (2006)).

${ }^{16}$ See Lemma 3 in the appendix for a formal proof.

${ }^{17}$ Note then all agents in the skilled sector match with the first partner that they encounter.

${ }^{18}$ That is, for $\theta$ marginally below $R_{1}$ we have $a\left(\theta, \theta^{\prime}\right)=0$ for $\theta^{\prime} \in \Theta_{1}$ and $a\left(\theta, \theta^{\prime}\right)=1$ otherwise.
} 
conclude that the matching market features $K$ classes. ${ }^{19}$ Unlike models in which $\hat{\theta}$ is exogenous, here it is necessary to compute $R_{K}$ even when $R_{K}<\hat{\theta}$ since this pins down the (off-equilibrium) consequences of investing for non-investors. That is, those with types in $\left[R_{K}, \hat{\theta}\right)$ anticipate that if they were to invest they would be acceptable to class $K$.

Figure 1 depicts the (first few) acceptance thresholds $R_{k}$ as a function of the marginal type $\hat{\theta}$ for parameter values $\{\alpha=1, r=0.1\}$ when types are uniformly distributed.

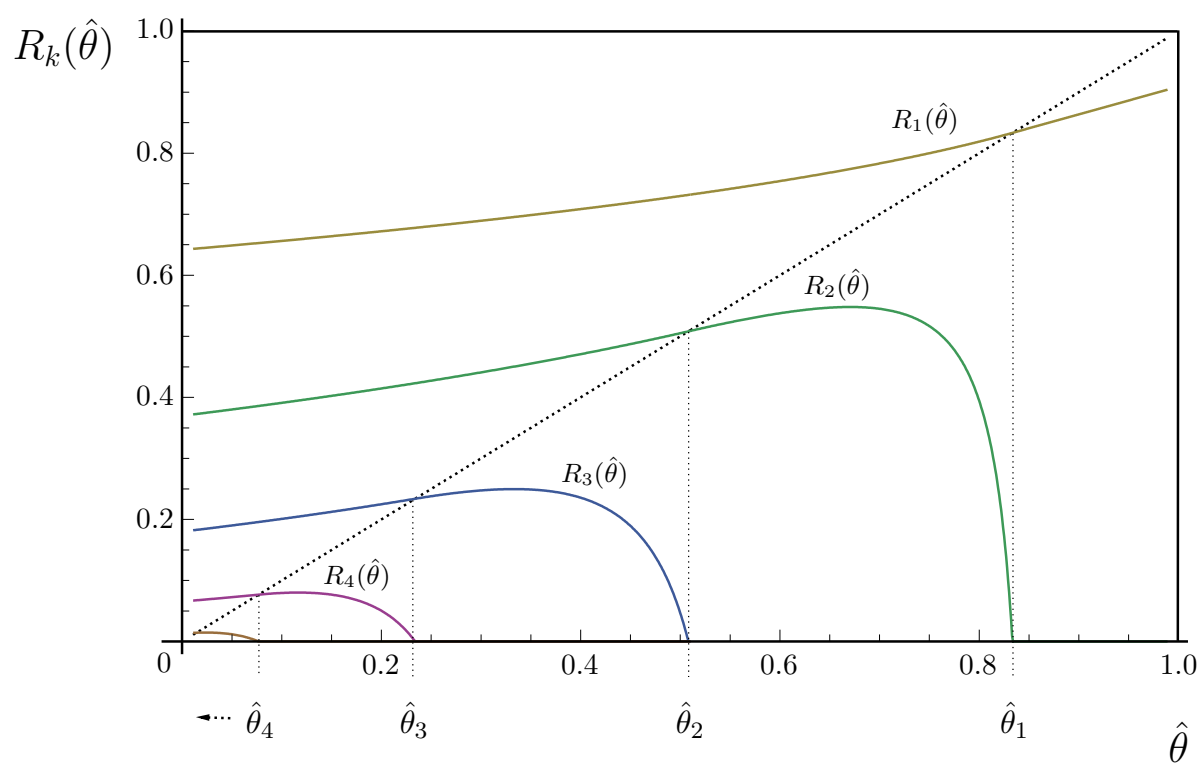

Figure 1: Determining $R_{k}$.

First consider $R_{1}$. For $\hat{\theta}$ very close to one, the worst type in the market is not too different from the best type. So all agents in the market find it optimal to accept the first partner they encounter, and $R_{1}<\hat{\theta}$ in this region. As $\hat{\theta}$ decreases, i.e. lower types start to enter, the expected payoff to continued search drops. Agents in the first class become increasingly willing to accept lower types; $R_{1}$ falls with $\hat{\theta}$. At the point $\hat{\theta}_{1}$ - a fixed point of $R_{1}$ - all agents in the market are indifferent between accepting a match with the lowest type (present) and continued search. As $\hat{\theta}$ falls below $\hat{\theta}_{1}$, a second class of agents emerges for now the lowest type in the market is no longer acceptable to all agents in the market. That is, $R_{1}>\hat{\theta}$ in this region. As agents of increasingly lower types enter 'effective' search frictions increase: class-1 agent never match with the new entrants workers. But these frictions do induce them to accept marginally lower types; that is, $R_{1}$ continues to decrease

\footnotetext{
${ }^{19}$ When $\hat{\theta}=0$ there are an infinite number of classes, but $K$ is necessarily finite when $\hat{\theta}>0$. See Result 8 in section A.3 of the appendix.
} 
with $\hat{\theta}$. The facts that $R_{1}$ has a unique fixed point $\hat{\theta}_{1} \in(0,1)$ and is strictly increasing, are general. ${ }^{20}$

Now turn to $R_{2}$. A second class only exists when $\hat{\theta}<\hat{\theta}_{1}$. When $\hat{\theta}$ is marginally below $\hat{\theta}_{1}$, the second class is very small so its members are unlikely to encounter someone willing to accept them. Their effective search frictions are very high, so they are willing to accept agents of very low types. Indeed, as $\hat{\theta}$ goes to $\hat{\theta}_{1}$ they become willing to accept anyone; $R_{2}$ goes to zero. As $\hat{\theta}$ falls from $\hat{\theta}_{1}$, class- 2 agents find continued search more attractive since the market becomes increasingly populated by match prospects. So $R_{2}$ initially increases but it is offset by an increasingly lower quality. At some point the value of continued search starts to fall, so $R_{2}$ also falls as $\hat{\theta}$ keeps decreasing. There comes a point, labeled $\hat{\theta}_{2}$, where agents are indifferent between accepting the marginal entrant and continuing search. Beyond this point a third class emerges and the same pattern repeats. The fact that each $R_{k}$ has a fixed point, $\hat{\theta}_{k}$, such that $R_{k}>\hat{\theta}$ (i.e. there are at least $k+1$ classes) for all $\hat{\theta}<\hat{\theta}_{k}$ is general, as is the fact that $\hat{\theta}_{k+1} \in\left(0, \hat{\theta}_{k}\right)$ and that $R_{k+1}$ approaches zero as $\hat{\theta}$ approaches $\hat{\theta}_{k} \cdot{ }^{21}$

\subsection{Endogenous Participation: Optimal Investment Decisions}

We now turn to the endogenous determination of the lowest type by considering the ex-ante investment problem. When costs are high enough, $c>\bar{c} \equiv(\alpha / r) /(r+\alpha)$, no agent finds it profitable to invest (even if they are the highest type and the matching pool consists only of the highest type). Any equilibrium outcome must feature no investment in this case. But, as in the static case, an equilibrium with no investment continues to exist when $c \leq \bar{c}$ because of a coordination problem. Again, such an equilibrium is not robust to deviations by groups of arbitrarily small measure. Specifically, by redefining

$$
u(\theta, \hat{\theta}) \equiv U(\theta \mid \hat{\theta})-c
$$

we can directly use the robustness concept from Definition 1. As in the static case, a no-investment equilibrium is robust when $c \geq \bar{c}$ and not when $c<\bar{c} .^{22}$ Henceforth we restrict attention to $c \leq \bar{c}$ and equilibria with positive investment. Our robustness concept will nevertheless continue to assist in equilibrium selection.

In general terms, an equilibrium in which the lowest type investing is $\hat{\theta} \in \Theta$ must satisfy (i) $U(\theta \mid \hat{\theta}) \geq c$ for all $\theta \geq \hat{\theta}$, and (ii) $U(\theta \mid \hat{\theta})<c$ for all $\theta<\hat{\theta}$. In order to derive equivalent, but simpler, conditions, we first compute the payoff $U(\theta \mid \hat{\theta})$ from investing using the bounds $R_{k}(\hat{\theta})$. Since $R_{1}=r U(\theta \mid \hat{\theta}) / \theta$ for all $\theta \in\left[R_{1}(\hat{\theta}), 1\right]$, and similarly for $k=2,3, \ldots, K, R_{k}=r U(\theta \mid \hat{\theta}) / \theta$ for

\footnotetext{
${ }^{20}$ See Lemmas 6 and 7 in the appendix.

${ }^{21}$ See Lemma 5 in the appendix.

${ }^{22}$ For a proof, see result 7 in section A.3 of the appendix.
} 
all $\theta \in\left[R_{k}(\hat{\theta}), R_{k-1}(\hat{\theta})\right)$,

$$
U(\theta \mid \hat{\theta})= \begin{cases}\theta \cdot R_{1}(\hat{\theta}) / r & \text { for all } \theta \in\left[R_{1}(\hat{\theta}), 1\right] \\ \theta \cdot R_{k}(\hat{\theta}) / r & \text { for all } \theta \in\left[R_{k}(\hat{\theta}), R_{k-1}(\hat{\theta})\right) \text { for } k=2,3, \ldots, K \\ 0 & \text { otherwise }\end{cases}
$$

Since $U(\theta \mid \hat{\theta})$ is weakly increasing in $\theta$, the two equilibrium conditions identified above become equivalent to

$$
U(\hat{\theta} \mid \hat{\theta}) \geq c
$$

and

$$
\lim _{\theta \uparrow \hat{\theta}} U(\theta \mid \hat{\theta}) \leq c
$$

The former ensures that all investing agents are acting optimally and the latter that all non-investing agents are acting optimally.

In analyzing (3.7), we note that since $\hat{\theta} \geq R_{K}(\hat{\theta})$ by definition, we have

$$
U(\hat{\theta} \mid \hat{\theta})=\frac{\hat{\theta} \cdot R_{K}(\hat{\theta})}{r}
$$

Returning to the uniform distribution illustration, Figure 2 shows $U(\hat{\theta} \mid \hat{\theta})$ for the same parameter values. We see that $U(\hat{\theta} \mid \hat{\theta})$ features discontinuities at each bound $\hat{\theta}_{k}$ that corresponds to a new class being formed. For $\hat{\theta} \in\left[\hat{\theta}_{1}, 1\right]$, there is only one class in equilibrium and therefore the payoff to the lowest type is increasing in the lowest type. However, for $\hat{\theta} \in\left[\hat{\theta}_{2}, \hat{\theta}_{1}\right)$ there are two classes in equilibrium and the payoff is non-monotonic in the lowest type. Intuitively, the payoff to the lowest type decreases in the lowest type as $\hat{\theta}$ approaches $\hat{\theta}_{1}$ since it becomes increasingly difficult for class two agents to find someone that is willing to accept them. This same pattern holds when there are $k$ classes, i.e. for $\hat{\theta} \in\left[\hat{\theta}_{k}, \hat{\theta}_{k-1}\right)$, for all $k \geq 2$.

In analyzing (3.8), we note that the weak inequality $R_{K}(\hat{\theta}) \leq \hat{\theta}$ implies that there are two cases to consider. First, if $R_{K}(\hat{\theta})<\hat{\theta}$ the function $U(\theta \mid \hat{\theta})$ is continuous in $\theta$ at $\theta=\hat{\theta}$ so that (3.8) is equivalent to $U(\hat{\theta} \mid \hat{\theta})=c$. In this type of equilibrium the marginal non-investor is dissuaded from investing because of cost considerations. This motivates the following.

Definition $2 A$ "cost constrained equilibrium" (CCE) is characterized by a cut-off type $\hat{\theta}$ that satisfies (i) $R_{K}(\hat{\theta})<\hat{\theta}$ and (ii) $U(\hat{\theta} \mid \hat{\theta})=c$.

The second possibility is that $R_{K}(\hat{\theta})=\hat{\theta}$, in which case the payoff $U(\theta \mid \hat{\theta})$ features a right jump discontinuity in $\theta$ at $\theta=\hat{\theta}$. Then we have $U(\hat{\theta} \mid \hat{\theta})=\hat{\theta}^{2} / r\left(\operatorname{since} R_{K}(\hat{\theta})=\hat{\theta}\right)$ and $U(\theta \mid \hat{\theta})=0$ for 


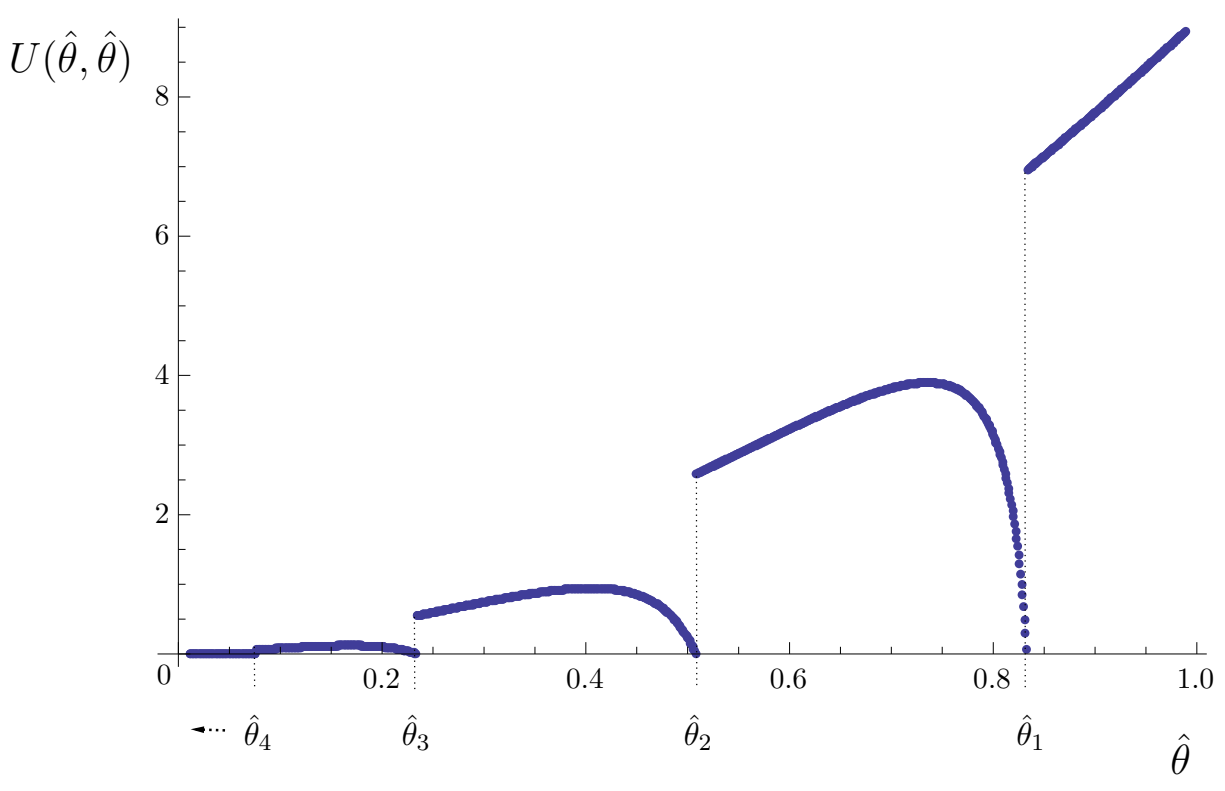

Figure 2: Determining $U(\hat{\theta} \mid \hat{\theta})$.

all $\theta<\hat{\theta}$. In this case (3.8) is automatically satisfied, and the marginal non-investor is deterred because they are never accepted as a partner. Thus

Definition 3 An "acceptance constrained equilibrium" (ACE) is characterized by a cut-off type $\hat{\theta}$ that satisfies (i) $R_{K}(\hat{\theta})=\hat{\theta}$ and (ii) $U(\hat{\theta} \mid \hat{\theta}) \geq c$.

With these definitions we can state our main results.

\subsection{Results}

We begin with results relating to the existence of robust equilibria, considering cost constrained equilibria and acceptance constrained equilibria in turn. Our first result indicates that cost constrained equilibria are relevant in the sense that there is a non-trivial set of parameters for which the unique robust equilibrium is cost constrained.

Proposition 2 There exists a non-empty open set of costs, $C^{c}$, such that for all $c \in C^{c}$, (i) a unique robust equilibrium exists, and (ii) it is a cost constrained equilibrium.

To get an intuition for this, and to highlight a range of related existence properties, consider Figure 3 in which we superimpose some possible investment costs onto a stylized version of Figure 2. Recalling definition 2 , we find the marginal types in cost-constrained equilibria at points at which $U(\hat{\theta} \mid \hat{\theta})$ equals $c$. For instance, at $c_{1}$ there is a cost constrained equilibrium with a marginal 
type of $\hat{\theta}_{c_{1}}$. This is the unique cost constrained equilibrium owing to the monotonicity of $U(\hat{\theta} \mid \hat{\theta})$ in this region. The same is of course true at any $c \in\left(U_{1}, \bar{c}\right)$, demonstrating that there is a unique cost constrained equilibrium for a non-trivial set of investment costs. Proposition 2 further establishes that these are the only equilibria in this region.

In contrast, there are multiple cost-constrained equilibria at a cost such as $c_{2}$, with corresponding marginal types of $\hat{\theta}_{c_{2}}$ and $\hat{\theta}_{c_{2}}^{\prime}$. Despite the multiplicity, only the former represents a robust equilibrium. To see this, note that if a group of agents with types marginally below $\hat{\theta}_{c_{2}}^{\prime}$ deviate by participating, then each will find that their resulting net payoff is positive regardless of how large the deviating group is. Furthermore, there are costs such as $c_{3}$ at which a unique, but non-robust, cost constrained equilibrium exists, and costs such as $c_{4}$ where three cost constrained equilibria exist where only one is robust.

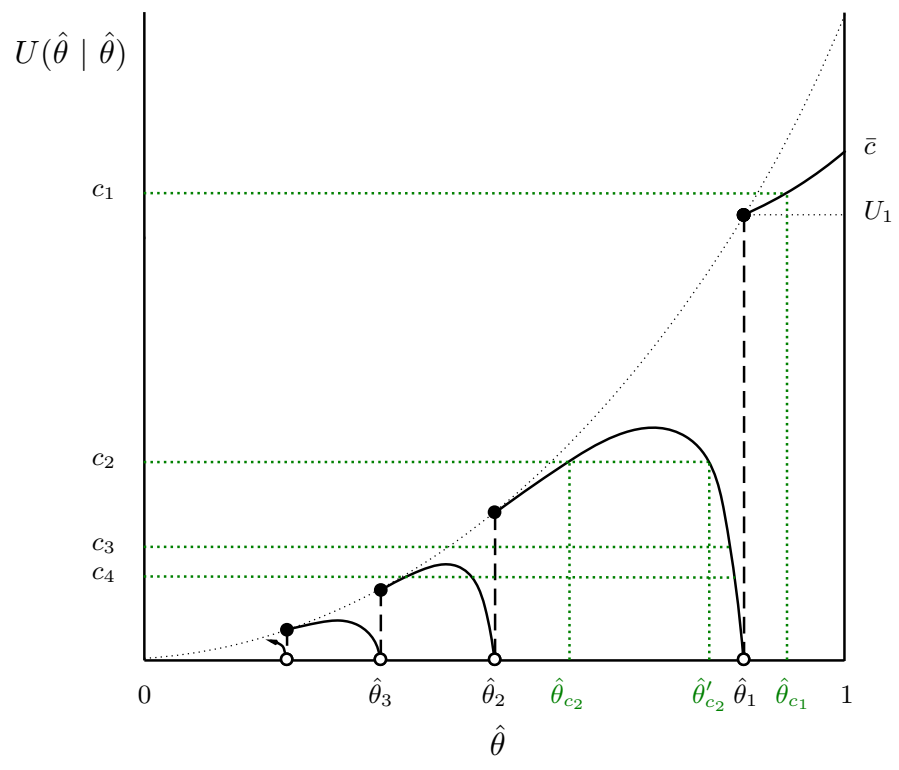

Figure 3: Existence of Cost Constrained Equilibria.

We now turn attention to the existence of acceptance constrained equilibria. In the same spirit as the preceding result, acceptance constrained equilibria are relevant in the sense that there is a non-trivial set of parameters for which the unique robust equilibrium is acceptance constrained.

Proposition 3 There exists a non-empty open set of costs, $C^{a}$, such that for all $c \in C^{a}$ (i) $a$ unique robust equilibrium exists, and (ii) it is an acceptance constrained equilibrium.

Unlike in the previous case, we are also able to demonstrate that acceptance constrained equilibria are also relevant in the sense that all the different possible 'varieties' of such equilibria exist for 
non-trivial sets of parameters. Specifically:

Proposition 4 For any $k \in\{1,2, \ldots\}$, there exists a non-empty open set of costs, $C_{k}$, such that a robust acceptance constrained equilibrium with $k$ classes exists for all $c \in C_{k}$.

To get some intuition and to highlight related existence properties, consider Figure 4. This figure is analogous to Figure 3, where we superimpose different investment costs. Recalling Definition 3, each $\hat{\theta}_{k}$ is a marginal type in an acceptance-constrained equilibrium as long as $c \leq U\left(\hat{\theta}_{k}, \hat{\theta}_{k}\right)$. For instance, at cost $c_{1}$ we have that $\hat{\theta}_{1}$ is the marginal type in an acceptance constrained equilibrium (with one class). This is of course true for any cost less than $U_{1}$. For any such cost the resulting acceptance constrained equilibrium is robust. At cost $c_{1}$ this is clear because there is no group of any size that could profitably deviate. ${ }^{23}$ At $\operatorname{cost} c_{2}$ or $c_{3}$, there is a profitable group deviation from the one-class ACE. However it requires that the group be sufficiently large: for sufficiently small groups (of positive size) the deviation is not profitable for all members of the deviating group. The robust acceptance constrained equilibrium is unique for $\operatorname{costs} c \in\left(U_{1}^{\prime}, U_{1}\right)$. This is the intuition underlying Proposition 3.

Similar reasoning can be applied to show that a robust acceptance constrained equilibrium with two classes exists at costs such as $c_{2}$ (any cost below $U_{2}$ will do), and with three classes at costs such as $c_{3}$, and so on. This is the intuition underlying Proposition 4 . The figure also illustrates

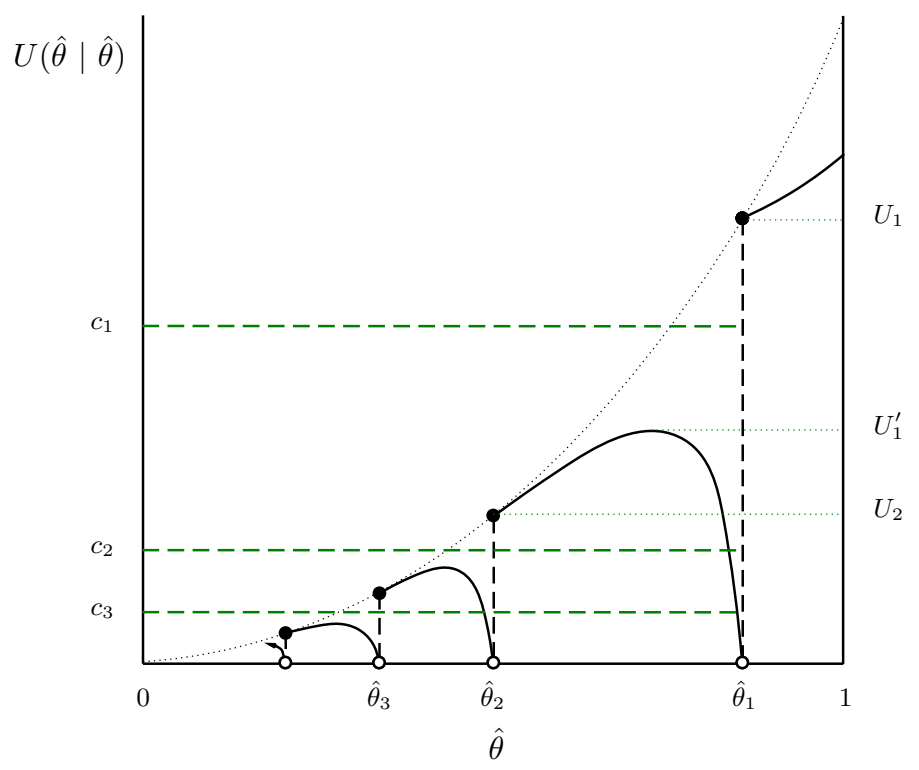

Figure 4: Existence of Acceptance Constrained Equilibria.

\footnotetext{
${ }^{23}$ To be sure, the highest payoff to the lowest deviating type is $U_{1}^{\prime}$, which is less than $c_{1}$.
} 
that multiple robust acceptance constrained equilibria can exist. For example at $\operatorname{cost} c_{2}$ a two-class and a one-class robust acceptance constrained equilibrium exists, and at cost $c_{3}$ a three-class robust acceptance constrained equilibrium is added to this list. Indeed,

Corollary 1 Multiplicity. If $c \in C_{k}$, then for each $k^{\prime} \in\{k, k-1, \ldots, 1\}$ there exists a robust acceptance constrained equilibrium with $k^{\prime}$ classes.

In addition to this, multiplicity of robust equilibria may arise because of the coexistence of acceptance constrained and cost constrained equilibria. For example, consider a cost $c_{4}$ in Figure 3. There are three robust equilibria: the CCE already discussed as well as a two-class ACE and, by Corollary 1, a one-class ACE.

Collecting the aforementioned results, a robust equilibrium always exists. ${ }^{24}$ There are two sources of multiplicity here. One is the discontinuity of $U(\hat{\theta} \mid \hat{\theta})$; this is the most important feature and is what generates the multiplicity of robust equilibria. The second source is the nonmonotonicity of $U(\hat{\theta} \mid \hat{\theta})$, which generates multiple CCE that are not robust.

Having established the existence of cost constrained and acceptance constrained equilibria, we now explore their properties.

Proposition 5 In a robust cost constrained equilibrium, participation is decreasing in $c$ and in frictions $1 / \alpha$.

These properties are analogous to those arising in the static benchmark, so we do not elaborate on them further here. Instead we explore the properties of robust ACE, which have counter-intuitive properties.

Proposition 6 In a robust acceptance constrained equilibrium, participation is unaffected by marginal changes in the investment cost, $c$, and is increasing in frictions, $1 / \alpha$.

The cost $c$ does not (locally) affect participation because in such equilibria the marginal noninvesting type would be willing to invest if they could secure the matching opportunities of even the lowest type in the matching market. That is, because of matching behaviour, the benefit of investing is too low (rather than the cost being too high as such). The second part of the Proposition comes from the fact that all participants become less 'choosy' as search frictions increase. As a result, some types that were previously excluded find that they are now acceptable to agents in the lowest class. Because they are strictly willing to pay the investment cost, such a change induces them to participate.

\footnotetext{
${ }^{24}$ A simple proof: (i) for $c \geq \bar{c}$, take a no-investment equilibrium, (ii) for $c \in\left[\hat{\theta}_{1}^{2} / r, \bar{c}\right.$ ) take the cost constrained equilibrium, and (iii) for $c<\hat{\theta}_{1}^{2} / r$ take the 1-class acceptance constrained equilibrium (of course, there are other possibilities).
} 
An implication of Proposition 6 is that, unlike in the static benchmark, there can be underinvestment in equilibrium. To see this, suppose that $c \in C_{1}$ so that a robust 1-class acceptance constrained equilibrium exists (e.g. in terms of figure 4 , any $c \in\left[0, U_{1}\right]$ ). If we consider lowering search frictions, Proposition 6 tells us that the marginal type in this equilibrium increases. Furthermore, the payoff of the marginal type ( $U_{1}$ in figure 4$)$ unambiguously increases-partners are found faster and are of a higher expected quality. As such, a robust acceptance constrained equilibrium continues to exist at the prevailing investment cost as frictions decrease. In fact, as frictions vanish the marginal participant in this equilibrium approaches the highest type. As such, in this equilibrium we have that participation vanishes to zero as frictions vanish. We do not offer a formal welfare analysis, but it is clear that arbitrarily small participation in the face of vanishing search frictions reflects under-investment. ${ }^{25}$

We now examine some more general properties not specific to either kind of equilibria. First we note that existence of robust equilibria implies that for any cost parameter there exists some type that is supported as the cut-off type in a robust equilibrium. However, it does not immediately implies that the opposite holds: i.e. for any type, does there exist some cost parameter that supports it as the cut-off type in a robust equilibrium. Understanding this speaks to models in which participation is fixed (e.g. Burdett and Coles (1997)); it determines whether an arbitrary level of participation corresponds to the post-investment stage of our model via the careful choice of $c$. This does not hold; furthermore, the set of types that cannot be supported (as the cut-off type) is not trivial.

Proposition 7 Non-arbitrary participation. There exists a non-empty open set of types that cannot be supported as the cut-off type of a robust equilibrium for any cost parameter.

Furthermore, the proof shows that this inability to support an arbitrary cut-off type is pervasive in the sense that such a non-empty set of types can be found within the interval $\left(\hat{\theta}_{k+1}, \hat{\theta}_{k}\right)$ for any $k \in\{1,2, \ldots\}$.

We conclude that search frictions have subtle effects on agents' incentives to participate. Specifically, a new class of equilibria (acceptance constrained) are shown to exist, to be robust (in the sense introduced in section 4.4), to be relevant (in the sense that the unique robust equilibrium is acceptance constrained for a non-negligible set of parameters), and to display comparative static

\footnotetext{
${ }^{25}$ From another perspective, we could hold search frictions fixed and consider reducing the investment cost to zero. Here the implication of Proposition 6 is that the participation level in this equilibrium is unaffected. The over-investment argument is less clear in this case however. Even if the investment cost is zero, it is not socially optimal for all agents to participate because participation imposes a congestion externality and lowers the payoff for existing participants. As such, it is possible that participation level in this equilibrium still reflects over-investment.
} 
properties that differ sharply from those arising in cost constrained equilibria. The fact that multiple robust equilibria arise implies that one can not generally identify whether participation in the skilled market is cost- or acceptance-constrained by simply looking at economic fundamentals such as investment costs, search frictions, or the distribution of ability. However, the comparative statics results may be informative in this regard-e.g. if participation is insensitive to investment cost, then this suggests that acceptance constraints are key.

\section{Multiple Search Markets}

Can robust acceptance constrained equilibria be eliminated by allowing for coordinated market segmentation? In exploring this we follow Jacquet and Tan (2007), who show how the existence of 'market-makers' leads to partition of the type space in equilibrium. More precisely, all agents within a partition enter the same marketplace and match with the first coworker they encounter. This 'perfect segmentation equilibrium' seems to address our coordination problem. As in the main analysis, it is instructive to derive the resulting partition for fixed participation, and then analyze the investment choice.

\subsection{The matching problem}

Consider marketplace 1 and suppose that for some $\theta_{1}$, types in $\left[\theta_{1}, 1\right]$ enter this marketplace; denote the conditional distribution of types in this marketplace by $G_{1}\left(\theta \mid \theta_{1}\right)$, defined in the usual way. The highest type accepts any coworker with type $\theta \geq M_{1}$ such that

$$
M_{1}=\frac{\alpha}{r} \cdot \int_{M_{1}}^{1}\left(\theta-M_{1}\right) d G_{1}\left(\theta \mid \theta_{1}\right) .
$$

Suppose there are at least two marketplaces, that is, $\hat{\theta}<\theta_{1}$. Then it must be that $M_{1}=\theta_{1}$ : the lowest accepted type is the marginal type in marketplace 1. From (4.1), $\theta_{1}$ must thus solve

$$
\theta_{1}=\frac{\alpha}{r} \cdot\left[\mathbb{E}\left[\theta \mid \theta \in\left[\theta_{1}, 1\right]\right]-\theta_{1}\right]
$$

The right-hand side of this starts at a positive value and is strictly decreasing in $\theta_{1}$ thanks to $\log$-concavity of $F$ (see section A.1); so a unique solution $\tilde{\theta}_{1}$, exists. Therefore $M_{1}=\theta_{1}=\tilde{\theta}_{1}$ for $\hat{\theta}<\tilde{\theta}_{1}$. On the other hand, if there is just one market-place $\hat{\theta}=\theta_{1}$. In this case $M_{1} \leq \hat{\theta}$, where, from (4.1), the value of $M_{1}$ satisfies

$$
M_{1}=\frac{\alpha}{r} \cdot\left[\mathbb{E}[\theta \mid \theta \in[\hat{\theta}, 1]]-M_{1}\right] .
$$


Simple re-arranging gives

$$
M_{1}=\frac{\alpha}{\alpha+r} \cdot \mathbb{E}[\theta \mid \theta \in[\hat{\theta}, 1]] \equiv m_{1}(\hat{\theta})
$$

which is indeed no greater that $\hat{\theta}$ if and only if $\hat{\theta} \geq \tilde{\theta}_{1} \cdot{ }^{26}$ To summarize, for $\hat{\theta} \in\left[0, \tilde{\theta}_{1}\right]$ the threshold $\theta_{1}=M_{1}=\tilde{\theta}_{1}$, and for $\hat{\theta} \in\left(\tilde{\theta}_{1}, 1\right]$ we have $\theta_{1}=\hat{\theta}$ and $M_{1}=m_{1}(\hat{\theta})$. More compactly, write: $\theta_{1}=\max \left\{\tilde{\theta}_{1}, \hat{\theta}\right\}$ and $M_{1}=\max \left\{\tilde{\theta}_{1}, m_{1}(\hat{\theta})\right\} .{ }^{27}$ Figure 5, which is analogous to Figure 1 , helps in picturing this.

If $\hat{\theta} \geq \tilde{\theta}_{1}$ we are done: all investors enter market 1 and accept the first partner they encounter. If $\hat{\theta}<\tilde{\theta}_{1}$, we construct the set of types entering the second marketplace in the same manner, except now the upper bound on types is $\theta_{1}$. The argument extends to define all the other boundaries. For $k \in\{2,3, \ldots\}$ let $\tilde{\theta}_{k}$ be the unique value that satisfies

$$
\tilde{\theta}_{k}=\frac{\alpha}{r} \cdot\left[\mathbb{E}\left[\theta \mid \theta \in\left[\tilde{\theta}_{k}, \tilde{\theta}_{k-1}\right)\right]-\tilde{\theta}_{k}\right]
$$

which defines a strictly decreasing sequence $\left\{\tilde{\theta}_{k}\right\}_{k}$ with a limit of zero. There are exactly $K$ classes if $\hat{\theta} \in\left[\tilde{\theta}_{K}, \tilde{\theta}_{K-1}\right)$, in which case $\theta_{k}=M_{k}=\tilde{\theta}_{k}$ for $k \in\{1, \ldots, K-1\}$ and $\theta_{K}=\hat{\theta}$ and $M_{K}=m_{K}(\hat{\theta})$ where

$$
m_{k}(\hat{\theta})=\frac{\alpha}{\alpha+r} \cdot \mathbb{E}\left[\theta \mid \theta \in\left[\hat{\theta}, \tilde{\theta}_{k-1}\right)\right]
$$

Figure 5 shows $M_{k}(\hat{\theta})$ in the case of the uniform distribution of types (see Appendix section C.2 for details). It differs from Figure 1 in two respects. First, to the left of the 45-degree line all cut-offs are constant. This is because there is no rejection once agents go to the right matching market. So once a class is fully formed its average quality is invariant in further entry; there is no crowding out. Second, the "off-equilibrium" acceptance threshold (to the right of the fixed-point) is increasing, rather than decreasing. Absent rejection the average quality of any one class increases when the marginal accepted type increases. These properties are not limited to a uniform distribution of types. We close with a useful Lemma.

Lemma 1 For each $k \in\{1,2, \ldots\}$, the function $M_{k}$ has a unique fixed point.

\footnotetext{
${ }^{26}$ To see this, we need $\hat{\theta}$ to be such that $m_{1}(\hat{\theta}) \leq \hat{\theta}$, which is the same as $(\alpha / r) \cdot[\mathbb{E}[\theta \mid \theta \in[\hat{\theta}, 1]]-\hat{\theta}]-\hat{\theta} \leq 0$. The expression on the left is decreasing in $\hat{\theta}$ and equals zero at $\tilde{\theta}_{1}$ (by definition).

${ }^{27}$ The expression for $\theta_{1}$ is obvious and the expression for $M_{1}$ holds because $m_{1}(\hat{\theta}) \geq \tilde{\theta}_{1}$ if and only if $\hat{\theta} \geq \tilde{\theta}_{1}$. To see this, $m_{1}(\hat{\theta}) \geq \tilde{\theta}_{1}$ is the same as $(\alpha / r) \cdot\left[\mathbb{E}[\theta \mid \theta \in[\hat{\theta}, 1]]-\tilde{\theta}_{1}\right] \geq \tilde{\theta}_{1}$, which holds if and only if $\hat{\theta} \geq \tilde{\theta}_{1}$ since the left side is increasing in $\hat{\theta}$ and equals $\tilde{\theta}_{1}$ when $\hat{\theta}=\tilde{\theta}_{1}$.
} 


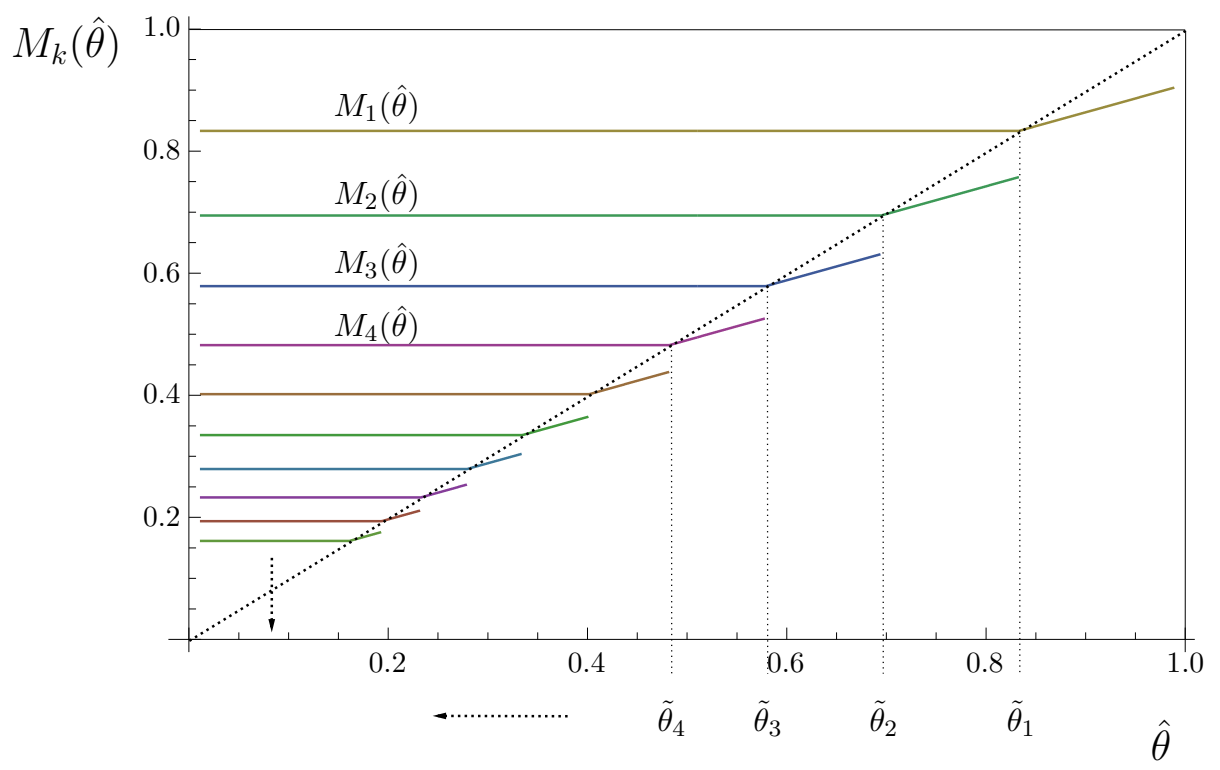

Figure 5: Determining $M_{k}(\hat{\theta})$ with Marketplaces.

\subsection{Endogenous Participation}

Let $M_{k}(\hat{\theta})$ denote the lowest acceptable type in market $k$ when the marginal investor is $\hat{\theta}$. For each type the value of entering the matching market is determined using the bounds $M_{k}(\hat{\theta})$ of the relevant class:

$$
U(\theta \mid \hat{\theta})= \begin{cases}\theta \cdot M_{1}(\hat{\theta}) / r & \text { if } \theta \in\left[M_{1}(\hat{\theta}), 1\right] \\ \theta \cdot M_{k}(\hat{\theta}) / r & \text { if } \theta \in\left[M_{k}(\hat{\theta}), M_{k-1}(\hat{\theta})\right) \text { for } k=2,3, \ldots, K \\ 0 & \text { otherwise. }\end{cases}
$$

An equilibrium is defined as in the one-marketplace case: for each type, investing must be profitable and a match must be acceptable. By continuity (within a class) and monotonicity of $U(\theta \mid \hat{\theta})$, it is sufficient to check these conditions for the lowest type entering. Therefore the same definitions of "cost constrained" and "acceptance constrained" equilibria apply (Definitions 2 and 3). It is helpful to characterize the gross payoff $U(\hat{\theta}, \hat{\theta})$ to the marginal investor.

Lemma $2 U(\hat{\theta}, \hat{\theta})$ is strictly increasing in $\hat{\theta}$, right-continuous, and displays a jump discontinuity at $\tilde{\theta}_{k}$ for $k \in\{1,2, \ldots\}$.

Here too each class bounds is associated with a discontinuity, however now the payoff to the lowest type is monotone. Figure 6 plots $U(\hat{\theta} \mid \hat{\theta})$ using the uniform distribution. As in Figure 2, the payoff 


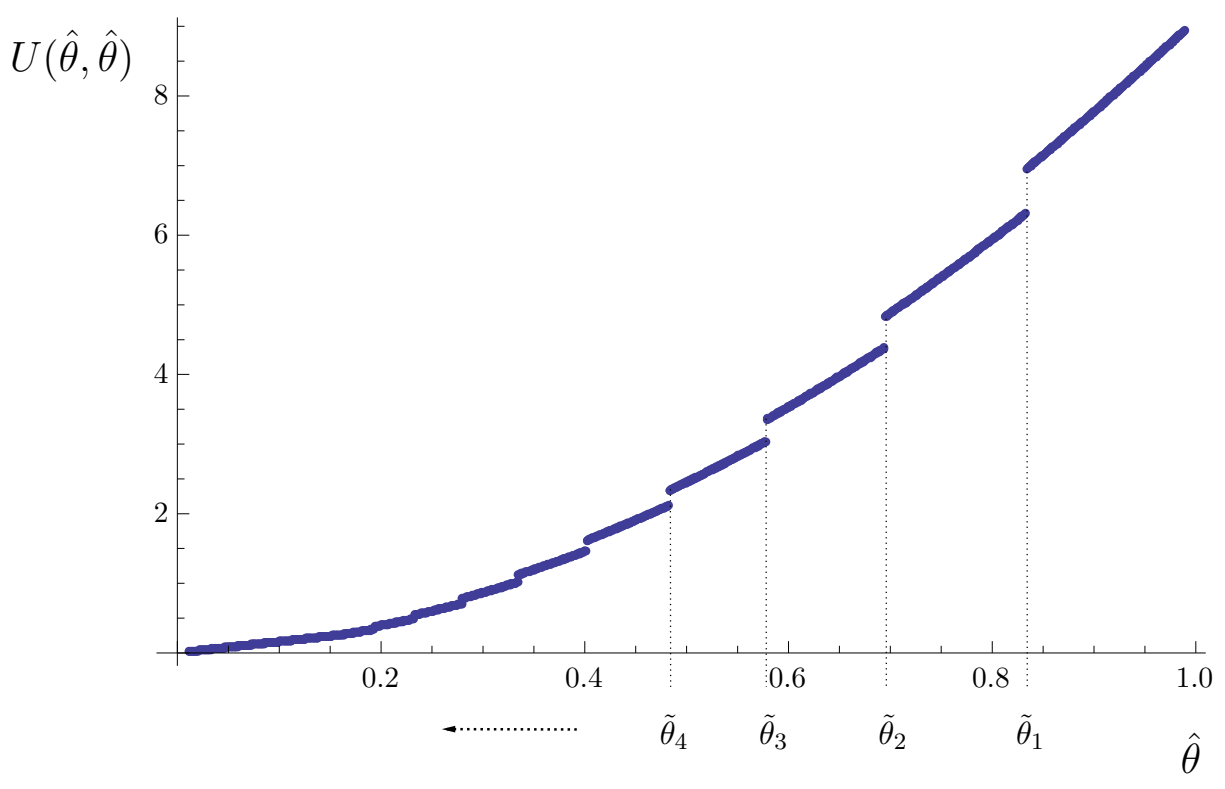

Figure 6: Determining $U(\hat{\theta} \mid \hat{\theta})$ with Marketplaces.

$U(\hat{\theta} \mid \hat{\theta})$ to the marginal entrant is discontinuous at each $\hat{\theta}_{k}$ because the highest type of the $k+1$ class necessarily matches with a lower (average) type than the average type in class $k$. But the local non-monotonicity of Figure 2 disappears because matching prospects cannot improve with entry; the matching probability within class is 1 (and 0 outside of one's class). So the payoff does not collapse to zero because matching within class is immediate, but the average quality decreases with more entrants. Thanks to this monotonicity it is sufficient that one other class- $k+1$ agent enters to form a match immediately. These properties are also general.

The most important implication of introducing these "horizontally differentiated" marketplaces is the elimination of multiple robust equilibria.

Proposition 8 A unique robust equilibrium exists for any $c<\bar{c} \equiv \alpha r /(r+\alpha)$.

This proposition has two main consequences. First, having multiple matching markets eliminates problems arising from multiplicity, including policy indeterminacy and the possibility of underinvestment identified earlier. Second, we can now be assured that any type can be supported as the cut-off type in the matching market via appropriate choice of $c$.

The intuition for 8 is clarified by comparing Figure 6 with Figure 2, and noting that it is the nonmonotonicity that is critical for the existence of multiple robust equilibria in the latter. Multiple cost constrained equilibria, robust or not, clearly relies on $U(\hat{\theta} \mid \hat{\theta})$ be non-monotone. Perhaps less obviously, this is also true for supporting multiple robust acceptance constrained equilibria. This 
is because the robustness of an acceptance constrained equilibrium requires that $U(\hat{\theta} \mid \hat{\theta})$ 'jumps' above $c$ at $\hat{\theta}_{k}$, and this can not occur more than once if $U(\hat{\theta} \mid \hat{\theta})$ is monotonic. Nevertheless, the fact that $U(\hat{\theta} \mid \hat{\theta})$ continues to display jump discontinuities in this setting implies that the our key qualitative results, relating to the existence and properties of robust acceptance constrained equilibria, continue to hold. Specifically:

Proposition 9 For each $k \in\{1,2, \ldots\}$ there exists a non-empty open set of costs such that the robust equilibrium is acceptance constrained with $k$ marketplaces. Similarly, for each $k \in\{1,2, \ldots\}$ there exists an open set of costs such that the robust equilibrium is cost constrained with $k$ marketplaces.

The intuition is straightforward if we were to superimpose investment costs onto Figure 6 . If the cost cuts $U(\hat{\theta} \mid \hat{\theta})$, then this identifies a robust cost constrained equilibrium. If it instead passes through a discontinuity, then this identifies a robust acceptance constrained equilibrium. ${ }^{28}$ In light of these results we also have natural extensions of Propositions 5 and 6.

Corollary 2 In the unique robust cost constrained equilibrium, participation is decreasing in the investment cost $c$ and in matching frictions $1 / \alpha$.

and

Corollary 3 In the unique robust acceptance constrained equilibrium with $k$ marketplaces, participation is insensitive to marginal changes in the investment cost $c$ and is increasing in matching frictions $1 / \alpha$.

\section{Discussion}

We discuss three assumptions of our model, and argue that they are not substantive for our main results.

Non-Transferable Utility. By assuming that agents simply consume their output, we are adopting a model with non-transferable utility (NTU). While this permits straightforward comparisons with much of the most closely related literature (e.g. Burdett and Coles (1997), Jacquet and Tan (2007), and Peters and Siow (2002)), we argue here that NTU is reasonable in our setting for a variety of reasons. Our leading application, the labor market, is usually cast in a two-sided setting

\footnotetext{
${ }^{28}$ To be sure, there may be also be non-robust ACE. For instance, if the cost is sufficiently low then there will be an ACE with a marginal type of $\tilde{\theta}_{1}$. It will not be robust however since an arbitrarily small set of deviating agents can invest and profitably join their separate market.
} 
in which matches are sought between firms and workers. In such settings, utility is assumed to be transferable since a firm pays a wage to their worker. In this model however matching only occurs between workers. The use of direct side-payments between coworkers is severely limited by social norms against the practice (consider co-authorship for instance), and in this respect the setting resembles a marriage problem. Even if side-payments were relatively costless to make, issues of contract incompleteness could easily render them prohibitively costly to enforce. For instance, an agent's 'output' should be more generally interpreted as 'utility' (e.g. a coworker makes a job easier) which is generally unverifiable private information. The relationship between workers would also be particularly susceptible to hold-up, given that a return to the matching market may be costly once a working relationship has been established.

It may be equally difficult for transfers between coworkers to be facilitated indirectly via a 'firm'. The firm may be bound by formal requirements, whether via legislation or collective bargaining, or by informal requirements grounded in 'fairness' or 'morale hazard' concerns (Fang and Moscarini (2005)), to pay workers similar amounts. Aside from such constraints, a firm will likely face significant informational asymmetries in terms of the nature and strength of the relationships between workers. Indeed, the workers may in fact be hired by completely different firms (as in co-authorship).

Even if all of the above frictions were somehow overcome, the fact that total output within the coworker pair is split evenly means that the model would be literally unchanged if side payments were permitted and determined by the Nash bargaining rule under the assumption that each agent has a threat point of zero (e.g. due to no production). As in Damiano and Li (2007), we opt for the more direct NTU specification in favor of setting up the bargaining problem explicitly.

Binary Investment Choice. We model the investment decision as a binary choice because it provides the simplest way to ensure heterogeneity among skilled agents (which is needed for the search problem to be interesting), and because it allows flexibility in interpreting 'investment' as an entry cost. Nevertheless, one tends to think of investment in less stark terms, envisioning a series of intermediate investment levels. As we outline in the appendix, our model can accommodate multiple investment levels and the results go through as long as the investment space remains discrete. However, the richer specification raises a number of other issues that are not our core focus here. For instance, there may be equilibria in which several investment levels arise within the same class, and it is not clear whether one should think of different investment levels as allowing entry into different matching markets. Such issues are certainly interesting, but we leave them to future research. 
The Clone Assumption. As in much of the related literature (e.g. Burdett and Coles (2001)), having agents that leave the market be replaced by agents with identical types is convenient because it immediately delivers a steady-state distribution of types in the matching market for any strategy profile. One alternative approach would be to impose an exogenous birth rate and use the distribution of types along with strategies to derive the steady state distribution of types in the matching market (e.g. as in Burdett and Coles (1997)). A second possibility is to impose an exogenous match destruction rate and have the matching pool being continually replenished by such displaced agents (as in Smith (2006)). In either case, it is known that block segregation continues to arise in equilibrium and therefore the discontinuities that underlie our main results remain. Furthermore, the clone assumption is particularly useful here because it helps isolate the source of multiplicity that we stress. For instance, even in the absence of skill investment it is known that multiple equilibria may arise when the steady state distribution of types is endogenously determined (e.g. Burdett and Coles (1999)). The adoption of the clone assumption makes it clear that the nature of multiplicity that we uncover is qualitatively distinct. Finally, we note that the clone assumption is completely innocuous in the extension with multiple matching markets by virtue of the fact that all agents match with the first partner they encounter.

\section{Conclusions}

We have studied a model in which agents make a costly investment in skill anticipating that skilled production involves productivity spillovers across coworkers. We show how the search for coworkers impinges on incentives to participate in the skilled market in somewhat subtle and sometimes counter-intuitive ways. Specifically, the search process does not simply lower the return to skill investment because it takes time to find a partner. Rather, we emphasize how search shapes the return to investing in skill by influencing the set of agents that are willing to accept the investor as a partner.

Analyzing how this 'indirect' effect of search plays out in equilibrium leads to a number of potentially policy-relevant results. For one, acceptance constrained equilibria emerge as a robust and relevant class of equilibria. Such equilibria possess comparative static properties that contrast sharply with the intuitive properties that arise in cost constrained equilibria-e.g. participation is unaffected by investment cost and is increasing in search frictions.

The analysis reveals a multiplicity of robust equilibria. These arise, in part, due to a coordination problem whereby marginal agents would participate if sufficiently many similar agents were to participate. Without such a coordinated deviation, each marginal agent correctly anticipates that they would not be accepted by anyone in the matching market, and is therefore dissuaded from 
participating. The fact that the deviating group needs to be sufficiently large underlies our notion of robustness.

This multiplicity is problematic-not only does it make the effect of policy unpredictable, we show it can lead to under-investment. However, we also show that multiplicity disappears once multiple matching markets are introduced as in Jacquet and Tan (2007). Nevertheless, robust acceptance constrained equilibria remain in this setting, suggesting that our main results are unaffected by the capacity for coordinated market segmentation by 'market-makers'. 


\section{Appendix}

\section{A Supporting Results}

\section{A.1 Log Concavity}

For our purposes, the key implication of a log concave density $f$ is the following. For any $\bar{x} \in \Theta$, let $\Psi:(-\infty, \bar{x}] \rightarrow \mathbb{R}$ be defined by

$$
\Psi(x \mid \bar{x}) \equiv \mathbb{E}[\theta \mid \theta \in[x, \bar{x}]]-x .
$$

Result 3 If $f$ is log concave, then $\Psi(x \mid \bar{x})$ is continuous and strictly decreasing in $x$.

The proof is omitted since the result is standard: e.g. see Bagnoli and Bergstrom (2005) and Kupka and Loo (1989).

\section{A.2 Robust Equilibria in Static Case}

The following results relate to claims made in the text about robust equilibria in the static case.

Result 4 In the static case, all no-investment equilibria are robust when $c \geq 1$.

Proof: Since there are no investors in the proposed equilibrium, we have $\tilde{\theta}=1$. For all $\varepsilon>0$ we have $u(1-\varepsilon, 1-\varepsilon)=(1-\varepsilon) \cdot \mathbb{E}[\theta \mid \theta \geq(1-\varepsilon)]-c<1-c \leq 0$.

Result 5 In the static case, none of the no-investment equilibria are robust when $c<1$.

Proof: Since there are no investors in the proposed equilibrium, we have $\tilde{\theta}=1$. For all $\varepsilon>0$ we have $u(1-\varepsilon, 1-\varepsilon)=(1-\varepsilon) \cdot \mathbb{E}[\theta \mid \theta \geq(1-\varepsilon)]-c \geq(1-\varepsilon) \cdot(1-\varepsilon)-c$. The final expression is a strictly decreasing continuous function of $\varepsilon$ that goes to $1-c>0$ as $\varepsilon \rightarrow 0$. There thus exists some $\bar{\varepsilon}>0$ such that it is strictly larger than zero for all smaller $\varepsilon$.

Result 6 In the static case, the unique equilibrium with positive investment is robust when $c \leq 1$.

Proof: Since $\hat{\theta}^{*} \leq 1$, we have $\tilde{\theta}=\hat{\theta}^{*}$ in the proposed equilibrium. For all $\varepsilon>0$ we have $u\left(\hat{\theta}^{*}-\right.$ $\left.\varepsilon, \hat{\theta}^{*}-\varepsilon\right)=\left(\hat{\theta}^{*}-\varepsilon\right) \cdot \mathbb{E}\left[\theta \mid \theta \geq\left(\hat{\theta}^{*}-\varepsilon\right)\right]-c<0$ (since the expression in (2.2) is strictly decreasing and equals zero at $\left.\hat{\theta}^{*}\right)$. 


\section{A.3 Fundamentals of the Dynamic Model}

The following is used to establish the claim that if a type is acceptable to some type, then they are acceptable to all lower types.

Lemma $3 R(\theta) \equiv \frac{r U_{\sigma}(\theta)}{\theta}$ is weakly increasing.

Proof: By dividing both sides of the asset value equation (3.1) by $\theta$, and slightly simplifying, we have:

$$
\begin{aligned}
R(\theta) & =\frac{\alpha}{r} \cdot \int_{0}^{1} a\left(\theta, \theta^{\prime}\right) \cdot \max \left\{\theta^{\prime}-R(\theta), 0\right\} d G\left(\theta^{\prime}\right) \\
& =\frac{\alpha}{r} \cdot \int_{R(\theta)}^{1} a\left(\theta, \theta^{\prime}\right) \cdot\left[\theta^{\prime}-R(\theta)\right] \cdot d G\left(\theta^{\prime}\right) .
\end{aligned}
$$

Consider two types, $\theta$ and $\tilde{\theta}>\theta$. A type $\theta^{\prime}$ will accept type $\tilde{\theta}$ whenever they accept a type $\theta$ (since $\left.\theta \geq R\left(\theta^{\prime}\right) \Rightarrow \tilde{\theta}>R\left(\theta^{\prime}\right)\right)$. Thus, $a\left(\tilde{\theta}, \theta^{\prime}\right) \geq a\left(\theta, \theta^{\prime}\right)$ for all $\theta^{\prime}$. Thus, for a fixed $R$, the right side of (A.1) is weakly increasing in $\theta$. Since the left side is strictly increasing in $R$ and the right side is strictly decreasing in $R$, it follows that $R(\tilde{\theta}) \geq R(\theta)$ (implicit function theorem).

Corollary 4 Equilibrium in the dynamic model must involve a cut-off type: $U_{\sigma}(\theta)$ is weakly increasing.

Proof: Use the fact that $U_{\sigma}(\theta)=R(\theta) \cdot \theta / r$ along with Lemma 3 .

Next we substantiate the claims made in the text about robust equilibria in the dynamic case.

Result 7 In the dynamic case, (i) all no-investment equilibria are robust when $c \geq \bar{c}$ and (ii) none of the no-investment equilibria are robust when $c<\bar{c}$.

Proof: Since there are no investors in the proposed equilibrium, we have $\tilde{\theta}=1$. For all for all $\varepsilon>0$ we have $U(1-\varepsilon, 1-\varepsilon)=(1-\varepsilon) \cdot R_{1}((1-\varepsilon)) / r \equiv \chi(\varepsilon)$. It is straightforward to see that $\chi$ is a continuous and strictly decreasing function with $\chi(0)=\bar{c}$. Part (i) follows since $\chi(\varepsilon)<\bar{c} \leq c$. Part (ii) follows since $\lim _{\varepsilon \rightarrow 0} \chi(\varepsilon)=\bar{c}>c$ implies that there exists some $\bar{\varepsilon}>0$ such that $\chi(\varepsilon$ ) is strictly larger than $c$ for all $\varepsilon<\bar{\varepsilon}$.

Finally, we prove the claim regarding the number of classes in equilibrium.

Result 8 Let $\hat{\theta} \in \Theta$ be the type of the marginal investor. There are an infinite number of classes if and only if $\hat{\theta}=0$. 
Proof: By replacing $\theta^{\prime}$ with the upper limit of integration, (3.6) implies

$$
R_{k} \leq \frac{\alpha}{r} \cdot \int_{R_{k}}^{R_{k-1}}\left[R_{k-1}-R_{k}\right] \cdot d G\left(\theta^{\prime} \mid \hat{\theta}\right) \leq \frac{\alpha}{r} \cdot\left[R_{k-1}-R_{k}\right]
$$

Simple rearranging then gives

$$
R_{k} \leq \gamma \cdot R_{k-1}
$$

where $\gamma \equiv \alpha /(\alpha+r)$. Returning to (3.6), noting that the right side is decreasing in $R_{k}$ and that $\gamma \in(0,1)$, equation (A.3) implies

$$
R_{k} \geq \frac{\alpha}{r} \cdot \int_{\gamma \cdot R_{k-1}}^{R_{k-1}}\left[\theta^{\prime}-\gamma \cdot R_{k-1}\right] \cdot d G\left(\theta^{\prime} \mid \hat{\theta}\right)>0 .
$$

To prove the "if" part, suppose that $\hat{\theta}=0$. If there are $k$ classes then, since $R_{k}>0=\hat{\theta}, R_{k+1}$ is well-defined and thus there are at least $k+1$ classes. By induction, there are an infinite number of classes in this case.

To prove the "only if" part, suppose that $\hat{\theta}>0$. Suppose to the contrary that $R_{k} \geq \hat{\theta}$ for all $k$ (i.e. that there are an infinite number of classes). An implication of (A.3) is

$$
R_{k} \leq \gamma^{k-1} \cdot R_{1}
$$

which together with (A.4) implies

$$
0<R_{k} \leq \gamma^{k-1} \cdot R_{1}
$$

Since $\gamma \in(0,1)$, the right side goes to zero as $k$ gets large. Since the upper and lower bounds on $R_{k}$ converge to zero, we have $\lim _{k \rightarrow \infty} R_{k}=0$. But this contradicts the supposition that $R_{k} \geq \hat{\theta}>0$ for all $k$.

\section{A.4 Identifying Critical Cut-Off Points}

The following lemmas provide the basis of Proposition 4, and use the facts (obvious from (3.6)) that $R_{k}$ is defined at each $\hat{\theta}$ such that $R_{k-1}(\hat{\theta})>\hat{\theta}$, and when defined, $R_{k}$ satisfies $R_{k}(\hat{\theta}) \in\left(0, R_{k-1}(\hat{\theta})\right)$, $R_{k}(\hat{\theta})$ is continuous, and $R_{k}(0)>0$ for all $k \in\{1,2, \ldots\}$.

Lemma 4 Let $\hat{\theta}^{*} \in(0,1)$ be a point such that, for some $k \in\{1,2, \ldots\}$, we have (i) $R_{k}\left(\hat{\theta}^{*}\right)=\hat{\theta}^{*}$, and (ii) $R_{k}(\hat{\theta})>\hat{\theta}$ for all $\hat{\theta} \in\left[0, \hat{\theta}^{*}\right)$. Then $\lim _{\hat{\theta}} \hat{\theta}^{*} R_{k+1}(\hat{\theta})<\hat{\theta}^{*}$.

Proof: By property (ii), $R_{k}$ and $R_{k+1}$ are defined and continuous functions on $\left[0, \hat{\theta}^{*}\right)$. Furthermore $R_{k}$ is continuous at $\hat{\theta}^{*}$ since $R_{k-1}\left(\hat{\theta}^{*}\right)>R_{k}\left(\hat{\theta}^{*}\right)=\hat{\theta}^{*}$, where the equality comes from property 
(i). Since $R_{k+1}(\hat{\theta})<R_{k}(\hat{\theta})$, we have that $\lim _{\hat{\theta} \nearrow \hat{\theta}^{*}} R_{k+1}(\hat{\theta}) \leq \lim _{\hat{\theta} \nearrow \hat{\theta}^{*}} R_{k}(\hat{\theta})=\hat{\theta}^{*}$, where the equality follows from the continuity of $R_{k}$ at $\hat{\theta}^{*}$. The weak inequality must be strict: if it were an equality the limits of integration on the right side of (3.6) would both converge to $\hat{\theta}^{*}$, implying that $\lim _{\hat{\theta} \uparrow \hat{\theta}^{*}} R_{k+1}(\hat{\theta})=0$. But then we would have $0=\lim _{\hat{\theta} \nearrow \hat{\theta}^{*}} R_{k}(\hat{\theta})=\hat{\theta}^{*}>0$, which is a contradiction.

Lemma 5 For each $k \in\{1,2, \ldots\}$ there exists a $\hat{\theta}_{k} \in(0,1)$ such that (i) $R_{k}\left(\hat{\theta}_{k}\right)=\hat{\theta_{k}}$, and (ii) $R_{k}(\hat{\theta})>\hat{\theta}$ for all $\theta \in\left[0, \hat{\theta}_{k}\right)$. Furthermore, $\hat{\theta}_{k+1}<\hat{\theta}_{k}$ and $\lim _{\hat{\theta} \nearrow \hat{\theta}_{k}} R_{k+1}(\hat{\theta})=0$.

Proof: We start with $\hat{\theta}_{1}$. Since $R_{1}$ is defined and continuous on [0,1], with $R_{1}(0)>0$ and $R_{1}(1)<1$, there exists at least one marginal type, $\hat{\theta}^{\prime} \in(0,1)$, such that $R_{1}\left(\hat{\theta}^{\prime}\right)=\hat{\theta}^{\prime}$. Define $\hat{\theta}_{1}$ to be the lowest such point (i.e. the first point at which $R_{1}$ touches the 45-degree line). By taking the lowest fixed point, note that we ensure that $R_{1}\left(\hat{\theta}_{1}\right)=\hat{\theta}_{1}$ and $R_{1}(\hat{\theta})>\hat{\theta}$ for all $\hat{\theta} \in\left[0, \hat{\theta}_{1}\right)$. Thus, the first part of the lemma holds for $k=1$ and $\hat{\theta}_{1}$ satisfies the conditions of lemma 4 .

Suppose that the first part of the lemma holds for $k$ : i.e. we have found a $\hat{\theta}_{k} \in(0,1)$ such that $R_{k}\left(\hat{\theta}_{k}\right)=\hat{\theta}_{k}$ and $R_{k}(\hat{\theta})>\hat{\theta}$ for all $\hat{\theta} \in\left[0, \hat{\theta}_{k}\right)$. Then $R_{k+1}(\hat{\theta})$ is defined and continuous on $\left[0, \hat{\theta}_{k}\right)$, with $R_{k+1}(0)>0$ and $\lim _{\hat{\theta}} \hat{\theta}_{k} R_{k+1}(\hat{\theta})<\hat{\theta}_{k}$ (the latter inequality follows from lemma 4). Therefore there exists some $\hat{\theta}^{\prime} \in\left(0, \hat{\theta}_{k}\right)$, such that $R_{k+1}\left(\hat{\theta}^{\prime}\right)=\hat{\theta}^{\prime}$. Again, define $\hat{\theta}_{k+1}$ be the lowest such point. Then we have found a $\hat{\theta}_{k+1} \in\left(0, \hat{\theta}_{k}\right)$ such that $R\left(\hat{\theta}_{k+1}\right)=\hat{\theta}_{k+1}$ and $R_{k+1}(\hat{\theta})>\hat{\theta}$ for all $\hat{\theta} \in\left[0, \hat{\theta}_{k+1}\right)$, so that the first part of the lemma holds for $k+1$ and $\hat{\theta}_{k+1}$ satisfies the conditions of lemma 4.

The proof of the first part of the lemma is complete by induction, since (i) we have shown that it holds for $k=1$, and (ii) have shown that it holds for $k+1$ if it holds for $k$.

As for the second part of the lemma, we have already shown (as part of the above construction) that $\hat{\theta}_{1} \in(0,1)$ and that $\hat{\theta}_{k+1} \in\left(0, \hat{\theta}_{k}\right)$, so that $\hat{\theta}_{k+1}<\hat{\theta}_{k}$ is ensured. To show the final claim, note that, since the integrand in (3.6) is positive only if $\theta \geq \hat{\theta}$, we can write

$$
\begin{aligned}
R_{k+1} & =\frac{\alpha}{r} \cdot \int_{\max \left\{R_{k+1}, \hat{\theta}\right\}}^{R_{k}}\left[\theta^{\prime}-R_{k+1}\right] \cdot d G\left(\theta^{\prime} \mid \hat{\theta}\right) \\
& =\frac{\alpha}{r} \cdot\left\{\int_{\hat{\theta}}^{R_{k}}\left[\theta-R_{k+1}\right] \cdot d G(\theta \mid \hat{\theta})-\mathbb{1}_{\left\{\hat{\theta}<R_{k+1}\right\}} \cdot \int_{\hat{\theta}}^{R_{k+1}}\left[\theta-R_{k+1}\right] \cdot d G(\theta \mid \hat{\theta})\right\},
\end{aligned}
$$

where $\mathbb{1}_{\left\{\hat{\theta}<R_{k+1}\right\}}$ is an indicator function that takes the value of one if $\hat{\theta}<R_{k+1}$ and zero otherwise. Now consider taking $\hat{\theta} \nearrow \hat{\theta}_{k}$. From lemma 4 it follows that $\lim _{\hat{\theta} \nearrow \hat{\theta}_{k}} \mathbb{1}_{\left\{\hat{\theta}<R_{k+1}(\hat{\theta})\right\}}=0$, and so the second term in the braces goes to zero as $\hat{\theta} \nearrow \hat{\theta}_{k}$. Both limits of integration in the first term in braces go to $\hat{\theta}_{k}$ as $\hat{\theta} \nearrow \hat{\theta}_{k}$, and therefore this term also goes to zero. We conclude then that the right side goes to zero as $\hat{\theta} \nearrow \hat{\theta}_{k}$, and thus $\lim _{\hat{\theta}} \hat{\theta}_{k} R_{k+1}(\hat{\theta})=0$ as required. 
Lemma $6 \quad R_{1}$ has a unique fixed point.

Proof: First note that $\hat{\theta}^{*}$ is a fixed point of $R_{1}$ if and only if it satisfies:

$$
\begin{aligned}
\hat{\theta}^{*} & =\frac{\alpha}{r} \cdot \int_{\hat{\theta}^{*}}^{1}\left(\theta-\hat{\theta}^{*}\right) \cdot d G\left(\theta \mid \hat{\theta}^{*}\right) \\
& =\frac{\alpha}{r} \cdot \int_{\hat{\theta}^{*}}^{1}\left(\theta-\hat{\theta}^{*}\right) \cdot \frac{f(\theta)}{1-F\left(\hat{\theta}^{*}\right)} \cdot d \theta \\
& =\frac{\alpha}{r} \cdot \frac{F(1)-F\left(\hat{\theta}^{*}\right)}{1-F\left(\hat{\theta}^{*}\right)} \cdot \int_{\hat{\theta}^{*}}^{1}\left(\theta-\hat{\theta}^{*}\right) \cdot \frac{f(\theta)}{F(1)-F\left(\hat{\theta}^{*}\right)} \cdot d \theta \\
& =\frac{\alpha}{r} \cdot\left[\mathbb{E}\left[\theta \mid \theta \in\left[\hat{\theta}^{*}, 1\right]\right]-\hat{\theta}^{*}\right] .
\end{aligned}
$$

The left side is strictly increasing in $\hat{\theta}^{*}$, starting from zero, and the right side is decreasing in $\hat{\theta}^{*}$ by assumption 3 , starting at a positive value and going to zero as $\hat{\theta}^{*} \rightarrow 1$. Therefore, there is a unique value of $\hat{\theta}^{*}$ that satisfies this.

On a side note, notice that the analogous expression for a fixed point of $R_{k}$ is

$$
\hat{\theta}^{*}=\frac{\alpha}{r} \cdot \frac{F\left(R_{k-1}\right)-F\left(\hat{\theta}^{*}\right)}{1-F\left(\hat{\theta}^{*}\right)} \cdot\left[\mathbb{E}\left[\theta \mid \theta \in\left[\hat{\theta}^{*}, R_{k-1}\right]-\hat{\theta}^{*}\right] .\right.
$$

If $R_{k-1}$ were a fixed value, then the same argument as the $k=1$ case would go through. But $R_{k-1}$ will be increasing in $\hat{\theta}^{*}$, and since the right side is increasing in $R_{k-1}$, we can not be sure that the right side is still decreasing in $\hat{\theta}^{*}$.

Corollary 5 There is exactly one class for all $\hat{\theta} \geq \hat{\theta}_{1}$.

Proof: We know that $R_{1}$ is defined on $[0,1]$, with $R_{1}(0)>0$ and $R_{1}(1)<1$, the fact that $\hat{\theta}_{1}$ is the unique fixed point (from lemma 6 ) implies that, $R_{1}(\hat{\theta}) \leq \hat{\theta}$ for all $\hat{\theta} \in\left[\hat{\theta}_{1}, 1\right]$.

Lemma $7 R_{1}$ is strictly increasing.

Proof: Since $R_{1}$ has a unique fixed point, we consider two cases. First, for $\hat{\theta} \leq \hat{\theta}_{1}$ we have that $R_{1}$ is defined by

$$
R_{1}=\frac{\alpha}{r} \cdot \int_{\hat{\theta}}^{1}\left(\theta-R_{1}\right) \cdot \frac{f(\theta)}{1-F(\hat{\theta})} \cdot d \theta .
$$

The left side is strictly increasing in $R_{1}$ whereas the right side is strictly decreasing in $R_{1}$ and strictly increasing in $\hat{\theta}$. Thus $R_{1}$ is strictly increasing in $\hat{\theta}$ by the implicit function theorem. Second, for $\hat{\theta} \geq \hat{\theta}_{1}$ we have that $R_{1}$ is defined by

$$
R_{1}=\frac{\alpha}{r} \cdot \int_{R_{1}}^{1}\left(\theta-R_{1}\right) \cdot \frac{f(\theta)}{1-F(\hat{\theta})} \cdot d \theta .
$$


The same argument goes through since the right side continues to be strictly decreasing in $R_{1}$ and strictly increasing in $\hat{\theta}$.

\section{A.5 Properties of $U(\hat{\theta} \mid \hat{\theta})$}

Corollary $6 U(\hat{\theta} \mid \hat{\theta})$ is discontinuous at each $\hat{\theta}_{k}$. Specifically,

$$
\lim _{\hat{\theta} \nearrow \hat{\theta}_{k}} U(\hat{\theta} \mid \hat{\theta})=0<U\left(\hat{\theta}_{k} \mid \hat{\theta}_{k}\right) .
$$

Proof: Follows since $U\left(\hat{\theta}_{k} \mid \hat{\theta}_{k}\right)=\hat{\theta}_{k}^{2} / r>0$ and

$$
\lim _{\hat{\theta} \nearrow \hat{\theta}_{k}} U(\hat{\theta} \mid \hat{\theta})=\lim _{\hat{\theta} \nearrow \hat{\theta}_{k}}\left\{\hat{\theta} \cdot R_{k+1}(\hat{\theta}) / r\right\}=\hat{\theta}_{k} \cdot\left\{\lim _{\hat{\theta} \nearrow \hat{\theta}_{k}} R_{k+1}(\hat{\theta})\right\} / r=\hat{\theta}_{k} \cdot 0 / r=0,
$$

where the second part of lemma 5 is used in the penultimate equality.

Lemma 8 The payoff to the marginal type is bounded above: $U(\hat{\theta} \mid \hat{\theta}) \leq \hat{\theta}^{2} / r$.

Proof: $U(\hat{\theta} \mid \hat{\theta})=\hat{\theta} \cdot R_{K}(\hat{\theta}) / r$ for some $K$. But $R_{K}(\hat{\theta}) \leq \hat{\theta}$ by definition of $K$. Thus $U(\hat{\theta} \mid \hat{\theta})=$ $\hat{\theta} \cdot R_{K}(\hat{\theta}) / r \leq \hat{\theta} \cdot \hat{\theta} / r$.

\section{B Proofs}

\section{Proof of Proposition 1.}

Proof: When $c<1$, (i) none of the 'no investment' equilibria are robust, while (ii) the unique equilibrium with investment is robust.

To prove (i), in a 'no investment' equilibrium $\tilde{\theta}=1$ and for all $\varepsilon>0$ we have $u(1-\varepsilon, 1-\varepsilon)=$ $(1-\varepsilon) \cdot \mathbb{E}[\theta \mid \theta \geq(1-\varepsilon)]-c$. This is a strictly decreasing continuous function of $\varepsilon$ that goes to $1-c>0$ as $\varepsilon \rightarrow 0$. Thus there exists some $\bar{\varepsilon}>0$ such that it is strictly larger than zero for all smaller $\varepsilon$.

To prove (ii), in the equilibrium with investment $\tilde{\theta}=\hat{\theta}^{*}$ and for all $\varepsilon>0$ we have $u\left(\hat{\theta}^{*}-\varepsilon, \hat{\theta}^{*}-\varepsilon\right)=\left(\hat{\theta}^{*}-\varepsilon\right) \cdot \mathbb{E}\left[\theta \mid \theta \geq\left(\hat{\theta}^{*}-\varepsilon\right)\right]-c<0$, where the strict inequality follows from noting that the expression in $(2.2)$ is strictly decreasing and equals zero at $\hat{\theta}^{*}$.

\section{Proof of Result 1.}

Proof: The left side of (2.2) is strictly increasing in $\hat{\theta}^{*}$ and strictly decreasing in $c$, while the right side is a constant. It follows (by the implicit function theorem) that $\hat{\theta}^{*}$ is increasing in $c$, and therefore participation is decreasing in $c$. 


\section{Proof of Result 2.}

Proof: Since $\frac{\partial}{\partial \hat{\theta}}\left(\mathbb{E}[\theta \mid \theta \geq \hat{\theta}]^{2}\right)=\frac{f(\hat{\theta})}{1-F(\hat{\theta})} \cdot 2 \cdot \mathbb{E}[\theta \mid \theta \geq \hat{\theta}] \cdot \Psi(\hat{\theta})$, where $\Psi(\hat{\theta}) \equiv \mathbb{E}[\theta \mid \theta \geq \hat{\theta}]-\hat{\theta}$, we have that marginal welfare is given by

$$
W^{\prime}(\hat{\theta})=f(\hat{\theta}) \cdot\{\mathbb{E}[\theta \mid \theta \geq \hat{\theta}](2 \cdot \Psi(\hat{\theta})-\mathbb{E}[\theta \mid \theta \geq \hat{\theta}])+c\}
$$

The term in braces is a continuous function of $\hat{\theta}$, approaching $\mathbb{E}[\theta]^{2}+c>0$ as $\hat{\theta} \rightarrow 0$ and $c-1<0$ as $\hat{\theta} \rightarrow 1$. Therefore there exists at least one $\hat{\theta}^{* *} \in(0,1)$ that satisfies the first-order condition $W^{\prime}\left(\hat{\theta}^{* *}\right)=0$.

To verify that such a point is unique and represents a maximum, observe that the term in braces is strictly decreasing in $\hat{\theta}$ at any such $\hat{\theta}^{* *}$. This follows from the fact that $\Psi^{\prime}(\hat{\theta})<0$ (from log concavity; see Result 3) implies the term in parentheses is strictly decreasing, along with the observation that the term in parentheses is necessarily negative at $\hat{\theta}^{* *}$ since $c>0$. Therefore $W^{\prime}(\hat{\theta})>0$ for $\hat{\theta}<\hat{\theta}^{* *}$ and $W^{\prime}(\hat{\theta})<0$ for $\hat{\theta}^{* *}<\hat{\theta}$. Therefore (i) there is a unique value that satisfies the first-order condition, and (ii) $W$ is pseudo-concave, implying that the first-order condition is sufficient for a maximum.

To show over-investment, use (2.2) to evaluate

$$
\begin{aligned}
\left.W^{\prime}(\hat{\theta})\right|_{\hat{\theta}=\theta^{*}} & =f\left(\theta^{*}\right) \cdot\left[\mathbb{E}\left[\theta \mid \theta \geq \theta^{*}\right]\left(2 \cdot \Psi\left(\theta^{*}\right)-\mathbb{E}\left[\theta \mid \theta \geq \theta^{*}\right]\right)+c\right] \\
& =f\left(\theta^{*}\right) \cdot\left[\mathbb{E}\left[\theta \mid \theta \geq \theta^{*}\right]\left(2 \cdot \Psi\left(\theta^{*}\right)-\mathbb{E}\left[\theta \mid \theta \geq \theta^{*}\right]\right)+\theta^{*} \mathbb{E}\left[\theta \mid \theta \geq \theta^{*}\right]\right] \\
& =f\left(\theta^{*}\right) \cdot \mathbb{E}\left[\theta \mid \theta \geq \theta^{*}\right]\left(\mathbb{E}\left[\theta \mid \theta \geq \theta^{*}\right]-\theta^{*}\right)>0 .
\end{aligned}
$$

This, along with the pseudo-concavity of $W$, implies that $\theta^{*}<\hat{\theta}^{* *}$.

\section{Proof of Proposition 2.}

Proof: By construction. Let $U_{1} \equiv U\left(\hat{\theta}_{1} \mid \hat{\theta}_{1}\right)=\hat{\theta}_{1}^{2} / r$, where $\hat{\theta}_{1}$ is that constructed in lemma 5 . From lemma 6 we easily deduce that $\hat{\theta}_{1}<\alpha /(\alpha+r)$. But then, $U_{1}<(\alpha /(r+\alpha))^{2} / r<(\alpha /(r+\alpha)) / r=\bar{c}$. Therefore $C^{c} \equiv\left(U_{1}, \bar{c}\right)$ is a non-empty open set. No ACE exists when $c \in C^{c}$. Suppose to the contrary that one did exist, with a cutoff of $\hat{\theta}^{*}$. Then $\hat{\theta}^{*} \leq \hat{\theta}_{1}$ by lemma 5 and therefore $U\left(\hat{\theta}^{*} \mid \hat{\theta}^{*}\right) \leq U_{1}$ by lemma 8 . But then $U\left(\hat{\theta}^{*} \mid \hat{\theta}^{*}\right) \leq U_{1}<c$, which contradicts $\hat{\theta}^{*}$ being an ACE.

Since $U(\hat{\theta} \mid \hat{\theta})=\hat{\theta} \cdot R_{1}(\hat{\theta}) / r$ for $\hat{\theta} \in\left[\hat{\theta}_{1}, 1\right]$ (by lemma 6 ) and $R_{1}(\hat{\theta})$ is strictly increasing and continuous on $[0,1]$, we have that $U(\hat{\theta} \mid \hat{\theta})$ is strictly increasing and continuous on $\hat{\theta} \in\left[\hat{\theta}_{1}, 1\right]$. Thus, for any $c \in C^{c}$ there exists a unique $\hat{\theta}^{c} \in\left(\hat{\theta}_{1}, 1\right)$ such that $U\left(\hat{\theta}^{c} \mid \hat{\theta}^{c}\right)=c, R_{1}\left(\hat{\theta}^{c}\right)<\hat{\theta}^{c}$, and $U(\hat{\theta} \mid \hat{\theta})$ cuts $c$ from below. Thus, there is a unique CCE, it has a cut-off of $\hat{\theta}^{c}$, and it is robust.

\section{Proof of Proposition 3.}


Proof: By construction. Let $C^{a} \equiv\left(\sup _{\hat{\theta}<\hat{\theta}_{1}} U(\hat{\theta} \mid \hat{\theta}), U\left(\hat{\theta}_{1} \mid \hat{\theta}_{1}\right)\right)$, where $\hat{\theta}_{1}$ is that identified in lemma 5.

We first show that $C^{a}$ is a well-defined open interval. First, note that for all $\hat{\theta}<\hat{\theta}_{1}$ we have:

$$
U(\hat{\theta} \mid \hat{\theta}) \leq \hat{\theta}^{2} / r<\hat{\theta}_{1}^{2} / r=U\left(\hat{\theta}_{1} \mid \hat{\theta}_{1}\right)
$$

The weak inequality follows from Lemma 8 , the strict inequality follows from $\hat{\theta}<\hat{\theta}_{1}$, and the equality follows by virtue of $R_{1}\left(\hat{\theta}_{1}\right)=\hat{\theta}_{1}$. Furthermore, by corollary 6 we have

$$
\lim _{\hat{\theta} \nearrow \hat{\theta}_{1}} U(\hat{\theta} \mid \hat{\theta})<U\left(\hat{\theta}_{1} \mid \hat{\theta}_{1}\right) .
$$

Together these imply that $\sup _{\hat{\theta}<\hat{\theta}_{1}} U(\hat{\theta} \mid \hat{\theta})<U\left(\hat{\theta}_{1} \mid \hat{\theta}_{1}\right)$ and, therefore, that $C^{a}$ is well-defined open set.

We then show that a unique equilibrium exists when $c \in C^{a}$, and that this equilibrium is robust and acceptance constrained.

First, no cost constrained equilibrium exists since there is no $\hat{\theta}$ such that $U(\hat{\theta}, \hat{\theta})=c \in C^{a}$ : all $\hat{\theta}<\hat{\theta}_{1}$ have $U(\hat{\theta}, \hat{\theta}) \leq \sup _{\hat{\theta}<\hat{\theta}_{1}} U(\hat{\theta} \mid \hat{\theta})$ by definition, and all $\hat{\theta} \geq \hat{\theta}_{1}$ have $U(\hat{\theta}, \hat{\theta}) \geq U\left(\hat{\theta}_{1}, \hat{\theta}_{1}\right)$ since $U(\hat{\theta} \mid \hat{\theta})$ is strictly increasing at $\hat{\theta} \in\left[\hat{\theta}_{1}, 1\right]$. The latter follows from $U(\hat{\theta} \mid \hat{\theta})=\hat{\theta} \cdot R_{1}(\hat{\theta}) / r$ for $\hat{\theta} \in\left[\hat{\theta}_{1}, 1\right]$ (by lemma 6 ) and $R_{1}(\hat{\theta})$ is strictly increasing.

Second, no acceptance constrained equilibrium with $k \geq 2$ classes exists. This is because $\hat{\theta}_{k}<\hat{\theta}_{1}$ by lemma 6 , and $U\left(\hat{\theta}_{k}, \hat{\theta}_{k}\right) \leq \sup _{\hat{\theta}<\hat{\theta}_{1}} U(\hat{\theta} \mid \hat{\theta})<c$.

Third, a unique acceptance constrained equilibrium with $k=1$ class exists: existence follows from $\hat{\theta}_{1}=R_{1}\left(\hat{\theta}_{1}\right)$ and $c \in C^{a} \Rightarrow c \leq U\left(\hat{\theta}_{1}, \hat{\theta}_{1}\right)$, and uniqueness follows from lemma 6 , since any $k=1$ class acceptance constrained equilibrium must have a cut-off of $\hat{\theta}_{1}$.

Finally, we show that this equilibrium is robust: by corollary $6, \lim _{\hat{\theta}} \nearrow \hat{\theta}_{1} U(\hat{\theta} \mid \hat{\theta})=0$, which implies that $u(\hat{\theta}, \hat{\theta})$ becomes strictly negative (goes to $-c$ ) for $\hat{\theta}$ marginally below $\hat{\theta}_{1}$.

\section{Proof of Proposition 4.}

Proof: For an ACE with $k$ classes, it must be that (i) there exists $\hat{\theta}$ such that $R_{k}(\hat{\theta})=\hat{\theta}$, and (ii) $U(\hat{\theta} \mid \hat{\theta}) \geq c$. Lemma 5 shows that for each $k \in\{1,2, \ldots\}$ there exists a cut-off value, $\hat{\theta}_{k} \in(0,1)$, such that $R_{k}\left(\hat{\theta}_{k}\right)=\hat{\theta}_{k}$. Thus $\hat{\theta}_{k}$ is a candidate cut-off value for an ACE with $k$ classes. Let $U_{k} \equiv U\left(\hat{\theta}_{k} \mid \hat{\theta}_{k}\right)=\left(\hat{\theta}_{k}\right)^{2} / r$, so that $\hat{\theta}_{k}$ is indeed an ACE cut-off for any $c \in C_{k} \equiv\left(0, U_{k}\right)$. The set $C_{k}$ is non-empty (since $\hat{\theta}_{k}>0$ implies $U_{k}>0$ ) and clearly open. Therefore an ACE equilibrium with $k$ classes exists for any $c \in C_{k}$. Note that $\lim _{\hat{\theta}} \hat{\theta}_{k} U(\hat{\theta} \mid \hat{\theta})=0$ by corollary 6 . Therefore an ACE with cut-off $\hat{\theta}_{k}$ is robust since $u(\hat{\theta}, \hat{\theta})$ is negative (goes to $-c$ ) for $\hat{\theta}$ marginally below $\hat{\theta}_{k}$. 


\section{Proof of Corollary 1.}

Proof: Follows from the observation that $C_{k} \subset C_{k^{\prime}}$ for all $k^{\prime} \in\{k, k-1, \ldots, 1\}$.

\section{Proof of Proposition 5.}

Proof: Let $\hat{\theta}^{*}$ be the cut-off associated with a robust cost constrained equilibrium. Then, $\hat{\theta}^{*}$ is defined by

$$
U\left(\hat{\theta}^{*} \mid \hat{\theta}^{*}\right)=c
$$

where $U$ cuts $c$ from below.

An increase in $c$ raises the right side of (B.2) without affecting the left side. Since the left side is increasing in $\hat{\theta}^{*}$, it follows that $\hat{\theta}^{*}$ is increasing in $c$. Thus, participation is decreasing in $c$ (implicit function theorem).

The left side of (B.2) equals $\hat{\theta}^{*} \cdot R_{K}\left(\hat{\theta}^{*}\right) / r$ for some $K$. Since $\partial R_{k} / \partial \alpha>0$ and $\partial R_{k} / \partial R_{k-1}>0$ for each $k$, it follows that $d R_{k} / d \alpha>0$ for each $k$. Therefore the left side is increasing in $\alpha$ and $\hat{\theta}^{*}$, whereas the right side is independent of $\alpha$. Therefore $\hat{\theta}^{*}$ is decreasing in $\alpha$ (implicit function theorem). That is, participation is decreasing in $1 / \alpha$.

Proof of Proposition 6: If $\hat{\theta}^{*}$ is the cut-off associated with a robust ACE, then for some $k \in\{1,2, \ldots\}$ we have

$$
R_{k}\left(\hat{\theta}^{*}\right)=\hat{\theta}^{*}
$$

and $U\left(\hat{\theta}^{*} \mid \hat{\theta}^{*}\right) \geq c$. Condition (B.3) is independent of $c$ and the latter condition holds for marginal changes in $c$.

An increase in $\alpha$ raises $R_{k}$ for a given $R_{k-1}$, and for $k \geq 2$ raises $R_{k-1}$ which further raises $R_{k}$. Since the ACE is robust, we have that $R_{k}$ cuts the 45-degree line from above. An increase in $R_{k}$ then implies that $\hat{\theta}^{*}$ must increase to restore condition (B.3). Therefore an increase in $\alpha$ raises the cutoff: i.e. a decrease in frictions lowers participation.

Proof of Proposition 7: A type, $\hat{\theta}$, cannot be supported as the cut-off type in a robust equilibrium if $U(\cdot \mid \cdot)$ is decreasing at $\hat{\theta}$. For any $k \in\{1,2, \ldots\}$, we know that there exists a greatest fixed point of $R_{k+1}$. Let this point be denoted $\hat{\theta}_{k}^{+}$, noting that $\hat{\theta}_{k}^{+} \in\left[\hat{\theta}_{k+1}, \hat{\theta}_{k}\right), U$ is continuous on $\left(\hat{\theta}_{k}^{+}, \hat{\theta}_{k}\right)$. Since $\lim _{\hat{\theta} \backslash \hat{\theta}_{k}^{+}} U(\hat{\theta} \mid \hat{\theta})=\left(\hat{\theta}_{k}^{+}\right)^{2} / r>0=\lim _{\hat{\theta}} \gamma \hat{\theta}_{k} U(\hat{\theta} \mid \hat{\theta})$, it follows that $U$ is decreasing on a non-negligible subset of $\left(\hat{\theta}_{k}^{+}, \hat{\theta}_{k}\right)$. Thus, there exists a non-empty open set of types, $\Theta_{k}^{*} \subseteq\left(\hat{\theta}_{k}^{+}, \hat{\theta}_{k}\right)$ such that $U(\cdot \mid \cdot)$ is strictly decreasing at each $\hat{\theta} \in \Theta_{k}^{*}$. 
Proof of Lemma 1: If $\theta_{k}^{*}$ is a fixed point of $M_{k}$ it satisfies (4.3). This has exactly one solution since the left side is increasing from zero in $\tilde{\theta}_{k}$ and the right side is strictly decreasing in $\tilde{\theta}_{k}$, starting at a positive value and going to zero as $\tilde{\theta}_{k} \rightarrow \tilde{\theta}_{k-1}$. (The RHS is the mean-residual life function.)

Proof of Lemma 2: Let $\tilde{\Theta}_{1} \equiv\left[\tilde{\theta}_{1}, 1\right]$ and for $k \geq 2$ let $\tilde{\Theta}_{k} \equiv\left[\tilde{\theta}_{k}, \tilde{\theta}_{k-1}\right)$. Then, for $\hat{\theta} \in \tilde{\Theta}_{k}$, we have

$$
U(\hat{\theta}, \hat{\theta})=\frac{\hat{\theta} \cdot M_{k}(\hat{\theta})}{r}=\frac{\alpha}{\alpha+r} \cdot \hat{\theta} \cdot \mathbb{E}\left[\theta \mid \theta \in\left[\hat{\theta}, \tilde{\theta}_{k-1}\right]\right]
$$

where $\tilde{\theta}_{0} \equiv 1 .^{29}$ Since $\tilde{\theta}_{k-1}$ is a constant, $U(\hat{\theta}, \hat{\theta})$ is clearly strictly increasing and right continuous on each $\tilde{\Theta}_{k}$. Since each $\hat{\theta}$ belongs to one and only one $\tilde{\Theta}_{k}$, it follows that $U$ is right continuous at each $\hat{\theta}$. Furthermore, from (B.4), we have

$$
\lim _{\hat{\theta} \uparrow \tilde{\theta}_{k}} U(\hat{\theta}, \hat{\theta})=\frac{\alpha}{\alpha+r} \cdot \tilde{\theta}_{k} \cdot \tilde{\theta}_{k}<\frac{\alpha}{\alpha+r} \cdot \tilde{\theta}_{k} \cdot \mathbb{E}\left[\theta \mid \theta \in\left[\tilde{\theta}_{k}, \tilde{\theta}_{k-1}\right]\right]=U\left(\tilde{\theta}_{k}, \tilde{\theta}_{k}\right)
$$

for each for $k \in\{1,2, \ldots\}$. There is an upward jump discontinuity at each $\tilde{\theta}_{k}$, and therefore the function is strictly increasing.

Proof of Proposition 8: Given that $U(\hat{\theta} \mid \hat{\theta})$ is strictly increasing, right-continuous, and ranges from 0 to $\bar{c}$, we have that $\hat{\theta}_{c} \equiv \min \left\{\hat{\theta} \in \Theta \mid U\left(\hat{\theta}_{c} \mid \hat{\theta}_{c}\right) \geq c\right\}$ is well-defined for any $c \in[0, \bar{c}]$. This value is a robust equilibrium cut-off since $U\left(\hat{\theta}_{c} \mid \hat{\theta}_{c}\right) \geq c$ (by construction) and $U\left(\hat{\theta}_{c}-\varepsilon \mid \hat{\theta}_{c}-\varepsilon\right)<c$ for all $\varepsilon>0$ (since $U$ is strictly increasing).

Uniqueness follows by contradiction: suppose that $\hat{\theta}^{\prime}$ and $\hat{\theta}^{\prime \prime}<\hat{\theta}^{\prime}$ were robust equilibrium cutoffs. The fact that $U$ is strictly increasing implies that, for $\varepsilon$ sufficiently small, we have $U\left(\hat{\theta}^{\prime \prime} \mid \hat{\theta}^{\prime \prime}\right)<$ $U\left(\hat{\theta}^{\prime}-\varepsilon \mid \hat{\theta}^{\prime}-\varepsilon\right)<c$, where the final inequality comes from the fact that $\hat{\theta}^{\prime}$ is a robust equilibrium cut-off. But then $\hat{\theta}^{\prime \prime}$ can not be a robust equilibrium cut-off since $U\left(\hat{\theta}^{\prime \prime} \mid \hat{\theta}^{\prime \prime}\right)<c$.

Proof of Proposition 9: For acceptance constrained equilibria, for each $k \in\{1,2, \ldots\}$ define $C_{k}^{A} \equiv\left(\lim _{\hat{\theta} \uparrow \tilde{\theta}_{k}} U(\hat{\theta} \mid \hat{\theta}), U\left(\tilde{\theta}_{k} \mid \tilde{\theta}_{k}\right)\right)$. This is a well-defined and open set (by Lemma 2). Take any $c \in C_{k}^{A}$ and note (i) $U\left(\tilde{\theta}_{k} \mid \tilde{\theta}_{k}\right) \geq c$ by construction, and (ii) $U\left(\tilde{\theta}_{k}-\varepsilon \mid \tilde{\theta}_{k}-\varepsilon\right) \leq \lim _{\hat{\theta} \uparrow \tilde{\theta}_{k}} U(\hat{\theta} \mid \hat{\theta})<c$ for all $\varepsilon>0$ (where the first inequality follows from $U$ being strictly increasing, and the second by construction of $C_{k}^{A}$ ). It follows that $\tilde{\theta}_{k}$ is a robust acceptance constrained equilibrium cut-off, which is unique by Proposition 8 .

For cost constrained equilibria, take $C_{k}^{C} \equiv\left(U\left(\tilde{\theta}_{k} \mid \tilde{\theta}_{k}\right), \lim _{\hat{\theta} \uparrow \tilde{\theta}_{k-1}} U(\hat{\theta} \mid \hat{\theta})\right)$. Since $U(\hat{\theta} \mid \hat{\theta})$ is continuous and strictly increasing at each $\hat{\theta} \in\left(\tilde{\theta}_{k}, \tilde{\theta}_{k-1}\right)$, for each $c \in C_{k}^{C}$ there exists a unique $\hat{\theta} \in\left(\tilde{\theta}_{k}, \tilde{\theta}_{k-1}\right)$ such that $U(\hat{\theta} \mid \hat{\theta})=c$. Since $\hat{\theta} \in\left(\tilde{\theta}_{k}, \tilde{\theta}_{k-1}\right)$, we have $M_{k}(\hat{\theta})<\hat{\theta}$, so that that $\hat{\theta}$ is a

\footnotetext{
${ }^{29}$ We opt to condition the expectation on a closed interval for notational convenience, but this is of course equivalent to conditioning on $\theta \in\left[\hat{\theta}, \tilde{\theta}_{k-1}\right)$ given that the type distribution is continuous.
} 
cost-constrained equilibrium with $k$ classes. Since $U$ is strictly increasing at this $\hat{\theta}$, this equilibrium is robust. It is unique by Proposition 8.

Proof of Corollary 2: Let $\hat{\theta}^{*}$ be the cut-off type in a CCE; it must satisfy $U\left(\hat{\theta}^{*} \mid \hat{\theta}^{*}\right)=c$. From robustness, the left side is increasing in $\hat{\theta}$ and independent of $c$. Since the right side is increasing in $c$, the implicit function theorem tells us $\hat{\theta}^{*}$ is increasing in $c$. So participation is decreasing in $c$.

Similarly, the right side is independent of $\alpha$ and the left side is increasing in $\alpha$ (both directly and because each $\tilde{\theta}_{k}$ increases in $\alpha$ ). Therefore $\hat{\theta}^{*}$ is decreasing in $\alpha$, thus participation is decreasing in $1 / \alpha$.

Proof of Corollary 3: Let $\hat{\theta}^{*}$ be the cut-off type in an ACE with $k$ classes. Then, it satisfies $M_{k}\left(\hat{\theta}^{*}\right)=\hat{\theta}^{*}$. That is, we have $\hat{\theta}=\tilde{\theta}_{k} \cdot{ }^{30}$ This condition is independent of $c$, and thus $\hat{\theta}^{*}$ (and therefore participation) is insensitive to marginal changes in $c$.

The left side is independent of $\alpha$ and the right side is increasing in $\alpha$ (because each $\tilde{\theta}_{k}$ increases in $\alpha$ ). Therefore $\hat{\theta}^{*}$ is increasing in $\alpha$, hence participation is increasing in $1 / \alpha$.

\section{Illustration Details (FOR ONLINE PUBLICATION)}

\section{C.1 Details of Illustration I}

When $F$ is uniform, we have

$$
G(\theta \mid \hat{\theta})= \begin{cases}1 & \text { for } \theta>1 \\ \frac{\theta-\hat{\theta}}{1-\hat{\theta}} & \text { for } \theta \in[\hat{\theta}, 1] \\ 0 & \text { otherwise }\end{cases}
$$

so that $R_{k}$ satisfies

$$
R_{k}=\frac{\alpha}{r} \cdot \int_{\max \left\{R_{k}, \hat{\theta}\right\}}^{R_{k-1}}\left[\frac{\theta^{\prime}-R_{k}}{1-\hat{\theta}}\right] \cdot d \theta^{\prime} .
$$

One can derive $R_{k}$ as an explicit function of $R_{k-1}$ by considering two cases.

If $R_{k} \geq \hat{\theta}$ then $R_{k}$ satisfies

$$
R_{k}=\frac{\alpha}{r} \cdot \int_{R_{k}}^{R_{k-1}}\left[\theta^{\prime}-R_{k}\right] \cdot d G\left(\theta^{\prime} \mid \hat{\theta}\right),
$$

\footnotetext{
${ }^{30} \mathrm{An}$ ACE also requires $U\left(\hat{\theta}^{*} \mid \hat{\theta}^{*}\right) \geq c$. In order for the statement of the proposition to be meaningful, we are implicitly only considering changes in parameters such that the inequality continues to hold. If the inequality is strict, then it will continue to hold when making marginal changes to parameters. In the special case where it holds with equality, we only consider marginal decreases in $c$ or $1 / \alpha$ (so that the inequality will continue to hold).
} 
which, by using the uniform functional form, gives

$$
2 \cdot \frac{r}{\alpha} \cdot(1-\hat{\theta}) \cdot R_{k}=\left(R_{k-1}-R_{k}\right)^{2},
$$

which, expressing $R_{k}$ as an explicit function of $R_{k-1}$, is:

$$
R_{k}=\frac{r}{\alpha} \cdot(1-\hat{\theta})+R_{k-1}-\sqrt{\frac{r}{\alpha} \cdot(1-\hat{\theta}) \cdot\left(\frac{r}{\alpha} \cdot(1-\hat{\theta})+2 \cdot R_{k-1}\right)} .
$$

Alternatively, if $R_{k}<\hat{\theta}$ then $R_{k}$ satisfies

$$
R_{k}=\frac{\alpha}{r} \cdot \int_{\hat{\theta}}^{R_{k-1}}\left[\theta^{\prime}-R_{k}\right] \cdot d G\left(\theta^{\prime}\right)
$$

which is

$$
2 \cdot \frac{r}{\alpha} \cdot(1-\hat{\theta}) \cdot R_{k}=R_{k-1}^{2}-\hat{\theta}^{2}-2 \cdot R_{k} \cdot\left(R_{k-1}-\hat{\theta}\right)
$$

so that

$$
R_{k}=\frac{1}{2} \cdot \frac{R_{k-1}^{2}-\hat{\theta}^{2}}{\frac{r}{\alpha} \cdot(1-\hat{\theta})+R_{k-1}-\hat{\theta}} .
$$

In calculating these boundaries, start with $k=1$ since $R_{1}$ is defined on the entire type space $[0,1]$. Then proceed to $k=2$, noting that $R_{2}$ is defined at the points for which $R_{1}>\hat{\theta}$. Successive values of $k$ can be used to compute $R_{k}$, noting that $R_{k}$ is defined at the points for which $R_{k-1}>\hat{\theta}$. The value of $K$ at $\hat{\theta}$ is that value of $k$ for which $R_{k} \leq \hat{\theta}$. The class boundaries are then $\theta_{k}=R_{k}$ for $k=1, \ldots, K-1$ and $\theta_{K}=\hat{\theta}$.

\section{C.2 Details of Illustration II}

Using the uniform distribution of type, we have that $\tilde{\theta}_{k}$ satisfies

$$
\tilde{\theta}_{k}=\frac{\alpha}{r} \cdot\left[\frac{\tilde{\theta}_{k-1}-\tilde{\theta}_{k}}{2}\right],
$$

which is

$$
\tilde{\theta}_{k}=\frac{1}{1+2 \cdot \frac{r}{\alpha}} \cdot \tilde{\theta}_{k-1}
$$

Using the initial condition that $\tilde{\theta}_{0}=1$, we have an explicit expression for $\tilde{\theta}_{k}$ :

$$
\tilde{\theta}_{k}=\left[\frac{1}{1+2 \cdot \frac{r}{\alpha}}\right]^{k} \text {. }
$$

Furthermore,

$$
m_{k}(\hat{\theta})=\frac{1}{1+\frac{r}{\alpha}} \cdot \frac{\hat{\theta}+\theta_{k-1}}{2} .
$$




\section{Elements of the Discussion (FOR ONLINE PUBLICATION)}

\section{D.1 More than two investment levels}

This section shows that our key results do not hinge on the binary investment assumption. Specifically, we show via a simple example how acceptance constrained equilibria continue to exist when we allow a large, but discrete, investment space. We also show how a richer investment space generates new features too; we leave a more comprehensive treatment to future research.

Suppose that agents can invest in discrete units so that $x_{i} \in\{0,1,2, \ldots\}$ and $s_{i}=x_{i} \cdot \theta_{i}$. The cost of $x$ units of investment is $c(x)$, which is strictly increasing. Consider an acceptance constrained equilibrium with one class in which all investors invest $x>0$. Given a cut-off type of $\hat{\theta}^{*}$, the asset value equation tells us that for $\theta \in\left[\hat{\theta}^{*}, 1\right], U\left(\theta \mid \hat{\theta}^{*}\right)$ satisfies:

$$
r U\left(\theta \mid \hat{\theta}^{*}\right)=\frac{\alpha}{r} \cdot\left[x \cdot \theta \cdot x \cdot \mathbb{E}\left[\theta^{\prime} \mid \theta^{\prime} \in\left[\hat{\theta}^{*}, 1\right]-r U\left(\theta \mid \hat{\theta}^{*}\right)\right]\right.
$$

A type $\theta^{\prime}$ will be accepted by a type $\theta \in\left[\hat{\theta}^{*}, 1\right]$ as long as $x \cdot \theta^{\prime} \cdot x \cdot \theta / r \geq U\left(\theta \mid \hat{\theta}^{*}\right)$, or

$$
\theta^{\prime} \geq R(\theta) \equiv r U\left(\theta \mid \hat{\theta}^{*}\right) /\left(x^{2} \cdot \theta\right)
$$

Using the asset value equation, this is:

$$
R(\theta)=\frac{\alpha}{r} \cdot\left[\mathbb{E}\left[\theta^{\prime} \mid \theta^{\prime} \in\left[\hat{\theta}^{*}, 1\right]-R(\theta)\right] .\right.
$$

Thus, as before, $R(\theta)$ is a constant for those in the first class. Furthermore, since $\hat{\theta}^{*}$ satisfies $R\left(\hat{\theta}^{*}\right)=\hat{\theta}^{*}$, it is straightforward to see that $\hat{\theta}^{*}=\hat{\theta}_{1}$ (where $\hat{\theta}_{1}$ is that used in the main analysis).

To produce closed-form expressions, suppose that the distribution of types is uniform on $[0,1]$. Assuming that a worker is accepted, their payoff when investing $x$ when others are investing $x^{*}$ is:

$$
U\left(\theta, x \mid \hat{\theta}_{1}, x^{*}\right)=\frac{1}{r} \cdot \frac{\alpha}{r+\alpha} \cdot x^{*} \cdot \frac{1+\hat{\theta}_{1}}{2} \cdot x \cdot \theta=Z \cdot x^{*} \cdot x \cdot \theta,
$$

where $Z \equiv \frac{1}{r} \cdot \frac{\alpha}{r+\alpha} \cdot \frac{1+\hat{\theta}_{1}}{2}$. For this to constitute an equilibrium a series of conditions must be met. First, all investors prefer investing $x$ to not investing (i.e. $x=0$ ). It is sufficient to verify that

$$
U\left(\hat{\theta}_{1}, x \mid \hat{\theta}_{1}, x\right) \geq c(x)
$$

which holds by making $c(x)$ small enough. Second, all investors prefer investing $x$ to any other level $x_{i}$. This implies two conditions: (i) that $0<x_{i}<x$ be deterred, and (ii) that $x_{i}>x$ be also deterred. For (i) a sufficient condition is that the highest type, when investing $x_{i}=x-1$, is not accepted in equilibrium. That is, $1 \cdot(x-1)<x \cdot \hat{\theta}_{1}$ (the left side is the skill of the highest type when 
they cut their investment by one step and the right is the minimum skill required for acceptance in equilibrium). This amounts to

$$
\hat{\theta}_{1}>1-(1 / x),
$$

which holds for $x$ low enough (of course, this always holds when $x=1$ ) and can be made to hold for any $x$ by setting $\alpha$ high enough. For (ii), it is sufficient to check that the highest types do not want to raise their investment, which holds when $c(x+1)$ sufficiently high:

$$
U\left(1, x+1 \mid \hat{\theta_{1}}, x\right)-c(x+1)<U\left(1, x \mid \hat{\theta_{1}}, x\right)-c(x)
$$

Last, all non-investors are acting optimally. As before, the fact that they would never be accepted ensures that non-investors do not want to invest $x$ (or less). The only remaining possibility is that some non-investors would profit by investing in excess of $x$, say $x+1$ (which necessarily gives those close to the marginal investor an acceptable skill level). To deter this one needs,

$$
U\left(\hat{\theta}_{1}, x+1 \mid \hat{\theta}_{1}, x\right)<c(x+1),
$$

which, again, is ensured by setting $c(x+1)$ high enough.

From this we see how even the simplest of acceptance constrained equilibria - one in which all investors invest the same amount and there is a single class - continue to exist in this setting. Richer forms of acceptance constrained equilibria are clearly possible, but here our intention is to highlight that it is the discreteness of investment possibilities that is important. We utilize the binary investment decision in our exposition for the simplicity it affords in illuminating the key mechanisms at play. For instance, even analyzing the case with three investment possibilities induces a new form of multiplicity whereby a one-class acceptance constrained equilibrium in which all investors choose $x=1$ coexists with one in which they invest $x=2$. To see this, note that the interesting constraints that must be satisfied are, (1) when $x=1$, a marginal non-investor does not want to invest $x_{i}=2$ to be accepted:

$$
Z \cdot 1 \cdot 2 \cdot \hat{\theta}_{1}-c(2)<0
$$

and (2) when $x=2$, a marginal investor wants to invest $x=2$ :

$$
Z \cdot 2 \cdot 2 \cdot \hat{\theta}_{1}-c(2)>0 \text {. }
$$

Clearly, a cost function such that

$$
c(2) \in\left(Z \cdot 2 \cdot \hat{\theta}_{1}, Z \cdot 4 \cdot \hat{\theta}_{1}\right)
$$

will satisfy both of these. The other constraints can be satisfied by taking $c(\cdot)$ such that $c(1)$ low enough, $c(3)$ large enough, and $\hat{\theta}_{1}$ such that $\hat{\theta}_{1}>1-(1 / 2)=1 / 2$ (this requires $\alpha>2 \cdot r$ with a uniform distribution). The source of this multiplicity is quite distinct from the sources we emphasize in the main model, and therefore leave a comprehensive treatment for future research. 


\section{References}

Acemoglu, D. and Angrist, J. (1999). How Large are the Social Returns to Education? Evidence from Compulsory Schooling Laws. NBER Working Papers 7444, National Bureau of Economic Research, Inc.

Anderson, S. and Bidner, C. (2013). Property Rights Over Marital Transfers. Tech. rep., University of New South Wales.

Autor, D. H., Levy, F. and Murnane, R. J. (2003). The skill content of recent technological change: An empirical exploration. The Quarterly Journal of Economics, 118 (4), 1279-1333.

Bagnoli, M. and Bergstrom, T. (2005). Log-concave probability and its applications. Economic Theory, 26 (2), 445-469.

Bidner, C. (2010). Pre-match investment with frictions. Games and Economic Behavior, 68 (1), 23 - 34.

- (2014). A spillover-based theory of credentialism. Canadian Journal of Economics, forthcoming.

Bloch, F. and Ryder, H. (2000). Two-sided search, marriages, and matchmakers. International Economic Review, 41 (1), 93-116.

Booth, A. and Coles, M. (2010). Education, matching, and the allocative value of romance. Journal of the European Economic Association, 8 (4), 744-775.

Burdett, K. and Coles, M. (1997). Marriage and class. The Quarterly Journal of Economics, 112 (1), $141-68$.

— and - (1999). Long-term partnership formation: Marriage and employment. Economic Journal, 109 (456), F307-34.

- and - (2001). Transplants and implants: the economics of self-improvement. The International Economic Review, $42(3), 597-616$.

Cole, H. L., Mailath, G. J. and Postlewaite, A. (2001). Efficient non-contractible investments in large economies. Journal of Economic Theory, 101 (2), 333-373.

Damiano, E. and Li, H. (2007). Price discrimination and efficient matching. Economic Theory, 30, $243-263$.

De Meza, D. and Lockwood, B. (2010). Too much investment? a problem of endogenous outside options. Games and Economic Behavior, 69 (2), 503-511.

Drago, R. and Garvey, G. T. (1998). Incentives for helping on the job: Theory and evidence. Journal of Labor Economics, 16 (1), 1-25.

FAnG, H. and Moscarini, G. (2005). Morale hazard. Journal of Monetary Economics, 52 (4), 749-777.

Felli, L. and Roberts, K. W. S. (2002). Does Competition Solve the Hold-up Problem? Tech. rep., C.E.P.R. Discussion Papers.

Gall, T., Legros, P. and Newman, A. (2006). The timing of education. Journal of the European Economic Association, 4 (2-3), 427-435. 
— - — and - (2012). Mismatch, rematch, and investment. Discussion Paper Series In Economics And Econometrics 189, Economics Division, School of Social Sciences, University of Southampton.

Gant, J., IChniowski, C. and Shaw, K. (2002). Social capital and organizational change in high-involvement and traditional work organizations. Journal of Economics and Management Strategy, 11 (2), 289-328.

Hatfield, J. W., Kojima, F. and Kominers, S. D. (2014). Investment incentives in labor market matching. American Economic Review: Papers and Proceedings, 104 (5), 436-41.

Hopkins, E. (2012). Job market signaling of relative position, or becker married to spence. Journal of the European Economic Association, 10 (2), 290-322.

Hoppe, H. C., Moldovanu, B. and Sela, A. (2009). The theory of assortative matching based on costly signals. The Review of Economic Studies, 76 (1), 253-281.

IChniowski, C. and Shaw, K. (2003). Beyond incentive pay: Insiders' estimates of the value of complementary human resource management practices. Journal of Economic Perspectives, 17 (1), 155-180.

Iyigun, M. and Walsh, R. P. (2007). Building the family nest: Premarital investments, marriage markets, and spousal allocations. The Review of Economic Studies, 74 (2), pp. 507-535.

JACQUET, N. L. and TAN, S. (2007). On the segmentation of markets. Journal of Political Economy, 115 (4), $639-664$.

Kupka, J. and Loo, S. (1989). The hazard and vitality measures of ageing. Journal of Applied Probability, 26(3), $532-542$.

Levy, F. and Murnane, R. J. (2004). The New Division of Labor. Princeton, New Jersey: Princeton University Press.

LuCAS, R. J. (1988). On the mechanics of economic development. Journal of Monetary Economics, 22 (1), 3-42.

Mailath, G. J., Postlewaite, A. and Samuelson, L. (2013a). Premuneration values and investments in matching markets, working paper, University of Pennsylvania.

—, - and - (2013b). Pricing and investments in matching markets. Theoretical Economics, 8 (2).

Moretti, E. (2004). Workers' education, spillovers, and productivity: Evidence from plant-level production functions. The American Economic Review, 94 (3), 656-690.

Murnane, R. J. and Levy, F. (1996). Teaching the New Basic Skills. New York: The Free Press.

Nöldeke, G. and Samuelson, L. (2014). Investment and Competitive Matching. Cowles Foundation Discussion Paper 1946, Yale University.

Peters, M. and Siow, A. (2002). Competing premarital investments. Journal of Political Economy, 110 (3), $592-$ 608.

SAnd, B. M. (2013). A re-examination of the social returns to education: Evidence from u.s. cities. Labour Economics, $24(0), 97-106$. 
Shimer, R. and Smith, L. (2000). Assortative matching and search. Econometrica, 68 (2), 343-370.

Smith, L. (2006). The marriage model with search frictions. Journal of Political Economy, 114 (6), 1124-1146.

Zhang, J. (1994). Bequest as a public good within marriage: A note. Journal of Political Economy, 102 (1), pp. $187-193$. 